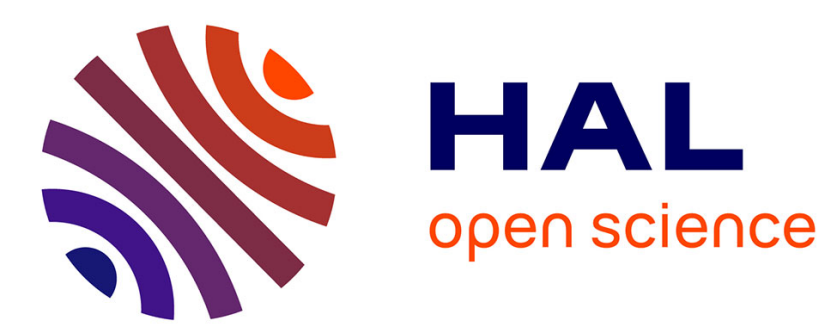

\title{
Nouvelle méthode de calcul de l'impédance de surface d'un métal dans le domaine infrarouge \\ J.C. Dudek
}

\section{To cite this version:}

J.C. Dudek. Nouvelle méthode de calcul de l'impédance de surface d'un métal dans le domaine infrarouge. Revue de Physique Appliquée, 1984, 19 (8), pp.573-592. 10.1051/rphysap:01984001908057300 . jpa-00245226

\section{HAL Id: jpa-00245226 https://hal.science/jpa-00245226}

Submitted on 1 Jan 1984

HAL is a multi-disciplinary open access archive for the deposit and dissemination of scientific research documents, whether they are published or not. The documents may come from teaching and research institutions in France or abroad, or from public or private research centers.
L'archive ouverte pluridisciplinaire HAL, est destinée au dépôt et à la diffusion de documents scientifiques de niveau recherche, publiés ou non, émanant des établissements d'enseignement et de recherche français ou étrangers, des laboratoires publics ou privés. 


\title{
REVUE DE PHYSIQUE APPLIQUÉE
}

Revue Phys. Appl. 19 (1984) 573-592

AOÛT 1984, PAGE 573

Classification

Physics Abstracts

$72.15 \mathrm{E}-72.30-78.20-78.70 \mathrm{G}$

\section{Nouvelle méthode de calcul de l'impédance de surface d'un métal dans le domaine infrarouge}

\author{
J. C. Dudek
}

Faculté des Sciences, BP 322, Abidjan 04, Côte d'Ivoire et Laboratoire de Physique du Vide et des Composants, CNAM, 292, rue Saint-Martin, 75141 Paris Cedex 03, France

(Reçu le 8 décembre 1983, révisé le 20 avril 1984, accepté le 2 mai 1984)

\begin{abstract}
Résumé. - Nous avons mis au point une méthode de calcul numérique de l'impédance de surface reposant sur les équations semi-classiques de la théorie de l'effet de peau anormal de Reuter et Sondheimer, dans le domaine infrarouge.

Notre méthode permet de calculer l'impédance de surface pour toute valeur du paramètre $p$ de diffusion $(0<p<1)$.

En outre, le principe de décomposition de l'onde électromagnétique dans le métal en un nombre discret $N$ d'ondes associées chacune à une direction particulière de déplacement des électrons de conduction permet de tenir compte aisément d'une anisotropie éventuelle du paramètre de diffusion. Aux longueurs d'ondes $\lambda$ inférieures à $200 \mu \mathrm{m}$, il suffit de prendre $N=4$ pour que l'écart relatif entre les résultats exacts obtenus à partir des séries de Dingle, valables seulement pour $p=0$ et $p=1$, et nos propres résultats ne dépasse pas $3 \times 10^{-2}$.
\end{abstract}

\begin{abstract}
We present here a numerical method for the calculation of the surface impedance $Z_{\mathrm{s}}$ of metals, based on the semiclassical equations of the anomalous skin effect theory by Reuter and Sondheimer. This method allows the calculation of $Z_{\mathrm{s}}$ for any value of the scattering parameter $p$ of conduction electrons hitting the surface and could easily account for a possible anisotropy of $p$. The electromagnetic wave in the metal is considered as the sum of a finite number $N$ of partial waves associated to discrete directions of the conduction electron momentum. For wavelengths inferior to $200 \mu \mathrm{m}$, it is sufficient to take $N$ as low as $N=4$ to obtain results in very good agreement with exact results calculated by using Dingle series in both cases $p=0$ and $p=1$.
\end{abstract}

\section{Introduction.}

La théorie de l'effet de peau anormal a été traitée en détail par Reuter et Sondheimer [1] dès 1948 pour une interface plane délimitant un métal semi-infini éclairé sous incidence normale. Les auteurs en ont déduit les expressions de l'impédance de surface $Z_{\mathrm{s}}$ pour les valeurs limites 0 et 1 du paramètre $p$ de diffusion des électrons de conduction.

Ces expressions se présentent sous formes d'intégrales peu commodes d'emploi que Dingle $[2,3]$ a réussi à développer en séries mieux adaptées au calcul numérique.

Toutefois, la méthode de Wiener et Hopf utilisée par Reuter et Sondheimer ne leur a pas permis de trouver de solution analytique pour $p$ quelconque.

Les tentatives effectuées récemment afin de calculer $Z_{\mathrm{s}}$ dans le cas général reposent sur des théories approchées $[4,5]$ nécessitant l'introduction d'hypothèses de nature empirique et conduisant par conséquent à des résultats non rigoureux bien que vraisemblables.

A notre connaissance, la théorie de $R$ et $S$ basée sur les équations de Boltzmann et Maxwell demeure la plus complète parmi toutes celles proposées dans le cadre semi-classique, dans la limite des approximations énoncées par ces auteurs (pages 337, 338 de [1]).

La méthode de calcul numérique que nous proposons au $\S 3$ en est l'application directe; elle repose sur 
une décomposition des champs électromagnétiques en ondes associées, chacune, aux électrons se déplaçant dans une direction faisant avec la surface un angle compris dans un intervalle $[\theta, \theta+\Delta \theta]$ donné. Si l'on considère $N$ directions différentes, on obtiendra $N+1$ composantes spectrales dans l'espace des vecteurs d'onde.

La comparaison des résultats ainsi obtenus aux valeurs calculées par les séries de Dingle pour $p=0$ ou 1 montre qu'il suffit de prendre $N$ égal à 4 pour obtenir une précision de l'ordre de 2 à $3 \%$ aux longueurs d'onde inférieures à $200 \mu \mathrm{m}$.

On a inclus dans les équations le terme de déplacement dû aux ions du réseau métallique; les résultats obtenus demeurent donc exacts aux courtes longueurs d'onde.

En outre, la décomposition minimale de $E_{x}(z)$ en deux ondes nous a permis de retouver la forme de l'expression approchée habituelle de la résistance de surface pour $\mathrm{p}$ quelconque :

$$
\operatorname{Re}\left\{Z_{\mathrm{s}}\right\} \# R_{\mathrm{cl}}\left[1+(1-p) \frac{3}{8} \frac{\omega_{\mathrm{p}}}{c} l\right]
$$

valable pour $\omega \tau \gg 1$, avec :

$R_{\mathrm{cl}}=\frac{1}{2 \omega_{\mathrm{p}} \tau} \sqrt{\frac{\mu_{0}}{\varepsilon_{0}}}:$ résistance de surface donnée par la $\omega_{\mathrm{p}}=\left(\frac{N e^{2}}{\varepsilon_{0} m^{*}}\right)^{1 / 2} \quad:$ pulsation de plasma du métal.

$l:$ libre parcours moyen des électrons de conduction.

Introduit initialement de manière empirique dans l'expression de l'absorption et apparaissant fréquemment dans les travaux relatifs aux propriétés optiques des couches métalliques [6,7], le facteur $(1-p)$ a été obtenu par Holstein [8] dans un calcul approché $(\tau \rightarrow \infty$ et champs variant exponentiellement dans le métal) basé sur des considérations énergétiques.

Le fait que notre méthode permette de retrouver ce facteur dans les mêmes conditions d'approximation que celles du calcul d'Holstein constitue un argument en sa faveur.

Une conséquence importante du principe de décomposition spectrale utilisé ici est que l'on peut calculer aisément la variation spatiale des champs au voisinage de la surface.

On constate alors qu'aux longueurs d'onde de quelques $\mu \mathrm{m}$, la loi de décroissance de l'amplitude du champ électrique reste très proche d'une exponentielle, quelle que soit l'importance de l'anomalie.

Cela justifie donc le fait que l'on puisse, dans le proche infrarouge, tenir compte de l'effet de peau anormal en introduisant dans l'expression de Drude de la constante diélectrique un temps de relaxation modifié $[9,10]$.
Enfin, nous proposons une méthode simple de calcul de $Z_{\mathrm{s}}$, pour un paramètre $p$ non isotrope, c'est-à-dire dépendant de l'incidence des électrons sur l'interface.

\section{Mise en équations.}

2.1. RAPPEL DE LA THÉORIE DE $R$ ET $S$. - Comme il est indiqué sur la figure 1, les champs électriques et magnétiques sont parallèles à $\mathrm{Ox}$ et $\mathrm{Oy}$ respectivement. L'interface est dans le plan $z=0$ et l'onde se propage parallèlement à l'axe $\mathrm{Oz}$ orienté positivement vers l'intérieur du métal.

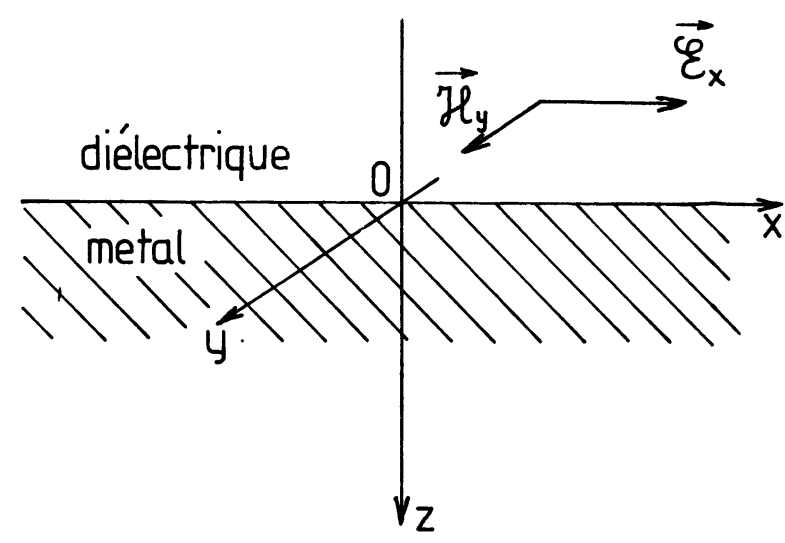

Fig. 1. - Interface métal-diélectrique. Système de coordonnée.

[Dielectric-metal interfaces : coordinates system.]

On cherche l'impédance de surface définie par :

$$
Z_{\mathrm{s}}=\frac{\mathcal{E}_{x}(z=0)}{\mathfrak{K}_{y}(z=0)} \text {. }
$$

Les champs sont supposés être monochromatiques et leurs valeurs instantanées sont les parties réelles des expressions :

$$
\begin{aligned}
\varepsilon_{x}(z, t) & =E_{x}(z) \exp (-i \omega t) \\
\mathcal{H}_{y}(z, t) & =H_{y}(z) \exp (-i \omega t) .
\end{aligned}
$$

Les équations de Maxwell permettent d'exprimer $Z_{\mathrm{s}}$ en fonction du champ électrique seul :

$$
Z_{\mathrm{s}}=i \omega \mu_{0} E_{x}(0)\left(\frac{\mathrm{d} E_{x}}{\mathrm{~d} z}\right)_{z=0^{+}}^{-1}
$$

$E_{x}(z)$ est solution du système intégro-différentiel suivant :

$$
\begin{gathered}
\frac{\partial F}{\partial z}+\left(\frac{1-i \omega \tau}{\tau v_{z}}\right) F=\frac{e}{m^{*} v_{z}} \frac{\partial f_{0}}{\partial v_{x}} E_{x} \\
\frac{\mathrm{d}^{2} E_{x}}{\mathrm{~d} z^{2}}+\varepsilon_{\mathrm{r}}\left(\frac{\omega}{c}\right)^{2} E_{x}=-i \omega \mu_{0} J_{x} \\
J_{x}=-2 e\left(\frac{m^{*}}{h}\right)^{3} \iiint v_{x} F \mathrm{~d} v_{x} \mathrm{~d} v_{y} \mathrm{~d} v_{z}
\end{gathered}
$$


(2) est l'équation de Boltzmann.

(3) résulte des équations de Maxwell, $\varepsilon_{\mathrm{r}}$ est la constante diélectrique du réseau métallique.

(4) est la densité de courant; elle ne possède qu'une composante suivant $\mathbf{O x}$ dans l'hypothèse d'un métal isotrope.

$m^{*}, \tau, e$ et $\left(v_{x}, v_{y}, v_{z}\right)$ sont respectivement la masse effective, le temps de relaxation, la charge et la vitesse des électrons de conduction dont la fonction de distribution peut s'écrire :

$$
f(\mathbf{v}, z)=f_{0}(|\mathbf{v}|)+\operatorname{Re}\{F(\mathbf{v}, z) \exp (-i \omega t)\} .
$$

Le $1^{\text {er }}$ terme du second membre est la distribution de Fermi :

avec :

$$
f_{0}(|\mathbf{v}|)=\left[1+\exp \left(\frac{w-w_{\mathrm{F}}}{k_{\mathrm{B}} T}\right)\right]^{-1}
$$

$$
\begin{aligned}
w & =\frac{1}{2} m^{*}|\mathbf{v}|^{2} \\
w_{\mathrm{F}} & =\text { énergie de Fermi } \\
k_{\mathrm{B}} & =\text { constante de Boltzmann } .
\end{aligned}
$$

Le $2^{\mathrm{e}}$ terme représente la perturbation due au champ électrique. Son amplitude complexe $F(\mathbf{v}, z)$ est à déterminer.

La solution générale de (2) est :

$$
\begin{gathered}
F(\mathrm{v}, z)=\mathrm{e}^{-\alpha z}\left[G(\mathrm{v})+\beta \int_{0}^{z} \mathrm{e}^{\alpha t} E_{x}(t)\right] \mathrm{d} t \\
\text { dans laquelle }\left\{\begin{array}{l}
\alpha=\frac{1-i \omega \tau}{\tau v_{z}} \\
\beta=\frac{e}{m^{*} v_{z}} \frac{\partial f_{0}}{\partial v_{x}} .
\end{array}\right.
\end{gathered}
$$

Les conditions aux limites sont incluses dans le terme $G(v)$. Il est commode de scinder les électrons de conduction en deux groupes caractérisés chacun par un signe de la composante $v_{z}$ de la vitesse.

On adoptera les conventions d'écriture suivantes :

$$
\begin{array}{c|r}
\mathbf{v}=v^{ \pm} & F(\mathbf{v}, z)=F^{ \pm}\left(\mathbf{v}^{ \pm}, z\right) \\
\alpha=\alpha^{ \pm} & G(\mathbf{v})=G^{ \pm}\left(\mathbf{v}^{ \pm}\right) \\
\beta=\beta^{ \pm} &
\end{array}
$$

Chaque grandeur étant affectée du signe + ou selon qu'elle appartient au groupe d'électrons se déplaçant respectivement avec une composante $v_{z}$ positive ou négative. $\mathbf{v}^{+}$et $\mathbf{v}^{-}$ne diffèrent que par le signe de $v_{z}$.

Les conditions aux limites s'écrivent alors :

$$
\begin{aligned}
& F^{+}\left(\mathbf{v}^{+}, z \rightarrow \infty\right)=0 \\
& F^{-}\left(\mathbf{v}^{-}, z \rightarrow \infty\right)=0 \\
& F^{+}\left(\mathbf{v}^{+}, z=0\right)=p F^{-}\left(\mathbf{v}^{-}, z=0\right)
\end{aligned}
$$

(6c) exprime le fait qu'une fraction $p$ des électrons de conduction est réfléchie spéculairement par l'interface.

$\mathrm{La}$ condition (6 a) est toujours vérifée car $\operatorname{Re}\left\{\alpha^{+}\right\}>0$.

La condition $(6 \mathrm{~b})$ donne :

$$
G^{-}\left(\mathrm{v}^{-}\right)=-\beta^{-} \int_{0}^{\infty} \mathrm{e}^{\alpha^{-} t} E_{x}(t) \mathrm{d} t
$$

d'où :

$$
F^{-}\left(\mathrm{v}^{-}, z\right)=-\beta^{-} \mathrm{e}^{-\alpha^{-z}} \int_{z}^{\infty} \mathrm{e}^{\alpha^{-} t} E_{x}(t) \mathrm{d} t .
$$

La condition (6 c) donne :

$$
G^{+}\left(\mathbf{v}^{+}\right)=-p \beta^{-} \int_{0}^{\infty} \mathrm{e}^{\alpha^{-t}} E_{x}(t) \mathrm{d} t
$$

d'où :

$$
F^{+}\left(\mathbf{v}^{+}, z\right)=\mathrm{e}^{-\alpha^{+} z}\left[-p \beta^{-} \int_{0}^{\infty} \mathrm{e}^{\alpha^{-} t} E_{x}(t) \mathrm{d} t+\beta^{+} \int_{0}^{z} \mathrm{e}^{\alpha^{+} t} E_{x}(t) \mathrm{d} t\right]
$$

Afin d'alléger l'écriture, on adoptera les conventions suivantes :

$$
\begin{aligned}
& \alpha \equiv \alpha^{+}=-\alpha^{-} \\
& \beta \equiv \beta^{+}=-\beta^{-} \\
& \left.\begin{array}{l}
\mathbf{v}^{+}=\left(v_{x}, v_{y}, v_{z}\right) \\
\mathbf{v}^{-}=\left(v_{x}, v_{y},-v_{z}\right)
\end{array}\right\} \text { avec } \quad v_{z}>0 \\
& E_{x} \equiv E \\
& H_{y} \equiv H \\
& J_{x} \equiv J
\end{aligned}
$$

La densité de courant s'écrira donc d'après (4) :

$$
J(z)=-2 e\left(\frac{m^{*}}{h}\right)^{3} \int_{-\infty}^{+\infty} \mathrm{d} v_{x} \int_{-\infty}^{+\infty} \mathrm{d} v_{y} \int_{0}^{\infty} v_{x} \beta \mathcal{F}\left(v_{z}, z\right) \mathrm{d} v_{z}
$$


avec :

$$
\mathcal{F}\left(v_{z}, z\right)=F^{+}\left(\mathrm{v}^{+}, z\right)+F^{-}\left(\mathrm{v}^{-}, z\right)=\int_{z}^{\infty} \mathrm{e}^{-\alpha(t-z)} E(t) \mathrm{d} t+\cdots+\int_{0}^{z} \mathrm{e}^{\alpha(t-z)} E(t) \mathrm{d} t+p \int_{0}^{\infty} \mathrm{e}^{-\alpha(t+z)} E(t) \mathrm{d} t
$$

Dans le système de coordonnées sphériques défini par la figure 2 on écrira :

$$
J(z)=-2 \frac{e^{2} m^{* 2}}{h^{3}} \int_{0}^{\pi / 2} \frac{\cos ^{3} \theta}{\sin \theta} \mathrm{d} \theta \int_{0}^{2 \pi} \cos ^{2} \varphi \mathrm{d} \varphi \int_{0}^{\infty} v^{2} \frac{\mathrm{d} f_{0}}{\mathrm{~d} v} \quad \mathcal{F}\left(v_{z}, z\right) \mathrm{d} v
$$

posons :

$$
s=\frac{v_{z}}{v}=\sin \theta
$$

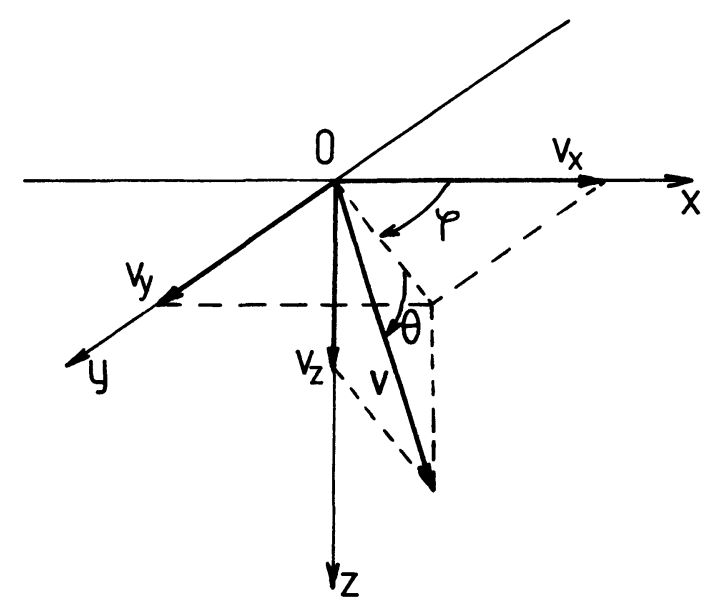

Fig. 2. - Système de coordonnées sphériques utilisé dans le demi-espace $z>0$.

[Spherical coordinates used in half-space $z>0$.]

Aux températures usuelles, on peut écrire :

$$
\frac{\mathrm{d} f_{0}}{\mathrm{~d} v} \#-\delta\left(v-v_{\mathrm{F}}\right)
$$

où $v_{\mathrm{F}}$ désigne la vitesse de Fermi.

En utilisant les relations :

$$
\begin{aligned}
& \sigma=\frac{N e^{2} \tau}{m^{*}}: \text { conductivité du métal massif. } \\
& N=\frac{8 \pi}{3} \frac{\left(m^{*} v_{\mathrm{F}}\right)^{3}}{h^{3}}: \text { densité d'électrons de conduction } .
\end{aligned}
$$

et :

$$
l=\tau v_{\mathbf{F}}
$$

on obtient finalement :

$$
J(z)=\frac{3}{4} \frac{\sigma}{l} \int_{0}^{1} \frac{1-s^{2}}{s} \mathrm{~d} s\left[\int_{z}^{\infty} \mathrm{e}^{-\alpha(t-z)} E(t) \mathrm{d} t+\int_{0}^{z} \mathrm{e}^{\alpha(t-z)} E(t) \mathrm{d} t+\cdots+p \int_{0}^{\infty} \mathrm{e}^{-\alpha(t+z)} E(t) \mathrm{d} t\right]
$$

$\alpha$ est une fonction de $s$ et a pour expression :

$$
\alpha=\frac{1-i \omega \tau}{l s}
$$


$E(z)$ est solution de l'équation de propagation (3) dans laquelle on a porté l'expression (7) du courant de conduction. A ce stade de la mise en équations, Reuter et Sondheimer ont cherché une solution analytique de $Z_{\mathbf{s}}$. Ils n'ont résolu le problème que pour $p=0$ et 1 , au prix de calculs assez laborieux pour $p=0$ (méthode de Wiener et Hopf).

Dans le présent travail, nous proposons une alternative à cette démarche, orientée d'emblée vers la recherche d'une méthode de résolution numérique.

L'expression (7) permet de faire quelques remarques concernant les limites de validité de l'expression locale classique du courant de conduction donnée par :

$$
J(z)=\frac{\sigma}{1-i \omega \tau} E(z) .
$$

Une façon d'exprimer la validité de cette expression est de supposer vérifiée l'inégalité :

$$
\left|\frac{1}{E(z)} \frac{\mathrm{d} E(z)}{\mathrm{d} z}\right| \ll|\alpha| .
$$

C'est-à-dire que la variation spatiale de $E(z)$ est beaucoup plus faible que celle des termes en $\mathrm{e}^{ \pm \alpha z}$.

On peut alors sortir le champ $E(z)$ des intégrales de (7) et écrire :

$$
J(z) \# \frac{3}{4} \frac{\sigma}{1-i \omega \tau} E(z) \int_{0}^{1}\left[2+(p-1) \mathrm{e}^{-\alpha z}\right]\left(1-s^{2}\right) \mathrm{d} s .
$$

A l'intérieur du métal, si $z \gg l$, le terme $\mathrm{e}^{-\alpha z}$ est négligeable devant l'unité et on retrouve bien l'expression locale (8) quel que soit $p$.

Par contre, au voisinage de la surface $(z \lesssim l),(10)$ n'est équivalente à (8) que si $p=1$.

Si $p \neq 1$, l'expression (8) n'est pas rigoureusement exacte même si la condition de « localité » (9) est satisfaite. Ce dernier point sera confirmé par l'allure des courbes $Z_{\mathrm{s}}(\lambda)$ obtenues aux courtes longueurs d'ondes.

On peut donner à (9) une forme plus explicite si l'on considère l'expression locale du champ :

$$
E(z)=E(0) \exp \left(i k_{\mathrm{m}} z\right)
$$

dans laquelle $k_{\mathrm{m}}=\varepsilon_{\mathrm{m}}^{1 / 2} \frac{\omega}{c}, \varepsilon_{\mathrm{m}}$ désignant la constante diélectrique de Drude du métal.

Dans les domaines visible et infrarouge, l'inégalité $\omega \ll \omega_{\mathrm{p}}$ permet d'écrire :

$$
\varepsilon_{\mathrm{m}} \# \frac{\sigma i}{\varepsilon_{0} \omega(1-i \omega \tau)} .
$$

Et la condition (9) devient :

$$
\frac{\omega}{c} \sqrt{\frac{\sigma}{\varepsilon_{0} \omega}} \frac{1}{\left(1+\omega^{2} \tau^{2}\right)^{1 / 4}} \ll \frac{\left(1+\omega^{2} \tau^{2}\right)^{1 / 2}}{l s} .
$$

Dans le cas le plus défavorable où $s=1$, on obtient :

$$
\frac{l}{\delta} \frac{\sqrt{2}}{\left(1+\omega^{2} \tau^{2}\right)^{3 / 4}} \ll 1
$$

avec $: \delta=\left(\frac{2}{\sigma \omega \mu_{0}}\right)^{1 / 2}:$ épaisseur de peau classique.

(11) exprime la condition qui doit être satisfaite pour que l'on puisse considérer le champ comme local avec toutefois les réserves exprimées plus haut lorsque $p \neq 1$. Elle est équivalente, à un facteur $\sqrt{2}$ près, à l'inégalité donnée par Reuter et Sondheimer (page 341 de [1]).

2.2 AUTRE FORME D'ÉCRITURE DES ÉQUATIONS DU PROBLÈME. - Dans l'expression (7), on distingue des termes en $\mathrm{e}^{-\alpha z}$ et $\mathrm{e}^{+\alpha z}$ correspondant respectivement à des ondes se propageant positivement et négativement le long de l'axe $\mathrm{Oz}$.

On peut donc définir une distribution de la densité de courant $j$ en fonction de la variable $s$ par :

$$
j(z, s)=\frac{\partial J}{\partial s}=j^{+}(z, s)+j^{-}(z, s)
$$


avec :

$$
\begin{aligned}
& j^{+}(z, s)=\frac{3}{4} \frac{\sigma}{l} \frac{1-s^{2}}{s}\left[\int_{0}^{z} \mathrm{e}^{\alpha t} E(t) \mathrm{d} t+p_{0} \int_{0}^{\infty} \mathrm{e}^{-\alpha t} E(t) \mathrm{d} t\right] \mathrm{e}^{-\alpha z} \\
& j^{-}(z, s)=\frac{3}{4} \frac{\sigma}{l} \frac{1-s^{2}}{s}\left[-\int_{0}^{z} \mathrm{e}^{-\alpha t} E(t) \mathrm{d} t+\int_{0}^{\infty} \mathrm{e}^{-\alpha t} E(t) \mathrm{d} t\right] \mathrm{e}^{\alpha z} .
\end{aligned}
$$

Remarque : Le paramètre de diffusion est maintenant noté $p_{0}$ afin de le distinguer de l'indice $p$ des variables discrètes du paragraphe suivant.

Dérivons ces expressions par rapport à $z$ :

$$
\begin{aligned}
& \frac{\partial j^{+}}{\partial z}=-\alpha j^{+}+A(s) E \\
& \frac{\partial j^{-}}{\partial z}=\alpha j^{-}-A(s) E
\end{aligned}
$$

avec :

$$
A(s)=\frac{3}{4} \frac{\sigma}{l} \frac{1-s^{2}}{s} .
$$

Combinant ces deux équations, on obtient :

$$
\frac{\partial^{2} j}{\partial z^{2}}+(i \alpha)^{2} j=-2 \alpha A(s) E .
$$

En outre, la forme des expressions (13) montre que l'on peut considérer la présence de $p_{0}$ comme résultant de la condition aux limites suivantes :

$$
j^{+}(z=0, s)=p_{0} j^{-}(z=0, s)
$$

soit encore :

$$
j(z=0, s)=\left(1+p_{0}\right) j^{-}(z=0, s) .
$$

Enfin, si à chaque composante différentielle $j(z, s) \mathrm{d} s$ du courant de conduction, on associe un champ électrique $\varepsilon(z, s) \mathrm{d} s$ tel que le champ électrique total soit donné par l'intégrale :

$$
E(z)=\int_{0}^{1} \varepsilon(z, s) \mathrm{d} s
$$

on peut alors réécrire l'équation de propagation (3) sous la forme suivante :

$$
\frac{\partial^{2} \varepsilon}{\partial z^{2}}+k_{\mathrm{r}}^{2} \varepsilon=-i \omega \mu_{0} j
$$

avec :

$$
k_{\mathrm{r}}^{2}=\varepsilon_{\mathrm{r}}\left(\frac{\omega}{c}\right)^{2} .
$$

La combinaison de (15) et (18) donne finalement l'équation de propagation unique :

$$
\left[\frac{\partial^{4}}{\partial z^{4}}+\left(k_{\mathrm{r}}^{2}-\alpha^{2}\right) \frac{\partial^{2}}{\partial z^{2}}-k_{\mathrm{r}}^{2} \alpha^{2}\right]\left(\begin{array}{c}
\varepsilon \\
\mathrm{ou} \\
j
\end{array}\right)=B(s) \int_{0}^{1}\left(\begin{array}{c}
\varepsilon \\
\mathrm{ou} \\
j
\end{array}\right) \mathrm{d} s
$$

dans laquelle :

$$
B(s)=\frac{3}{2} i \omega \mu_{0} \frac{\sigma}{l} \frac{1-s^{2}}{s} \alpha
$$

c'est la résolution numérique de cette équation que nous allons décrire. 


\section{Méthode de calcul.}

3.1 DisCRÉTISATION DES ÉQUATIONS, VECTEURS D'ONDE PROPRES. - On considère $N$ directions discrètes $\left\{s_{1}, s_{2}, \ldots, s_{N}\right\}$ appartenant à $N$ intervalles adjacents $\left\{\Delta_{1}, \Delta_{2}, \ldots, \Delta_{N}\right\}$ tels que $\sum_{n=1}^{N} \Delta_{n}=1$.

La discrétisation des variables $\varepsilon(z, s)$ et $j(z, s)$ consiste à leur faire correspondre les ensembles finis $\left\{E_{1}, E_{2}, \ldots, E_{N}\right\}$ et $\left\{J_{1}, J_{2}, \ldots, J_{N}\right\}$ tels que :

$$
\begin{aligned}
& E_{n}=\varepsilon\left(s_{n}\right) \Delta_{n} \\
& J_{n}=j\left(s_{n}\right) \Delta_{n}
\end{aligned}
$$

et par conséquent :

$$
\begin{aligned}
& J=\sum_{n=1}^{N} J_{n} \\
& E=\sum_{n=1}^{N} E_{n} .
\end{aligned}
$$

Les équations (18) et (19) deviennent donc :

$$
\begin{gathered}
{\left[\frac{\mathrm{d}^{2}}{\mathrm{~d} z^{2}}+k_{\mathrm{r}}^{2}\right] E_{n}=-i \omega \mu_{0} J_{n}} \\
{\left[\frac{\mathrm{d}^{4}}{\mathrm{~d} z^{4}}+\left(k_{\mathrm{r}}^{2}-\alpha_{n}^{2}\right) \frac{\mathrm{d}^{2}}{\mathrm{~d} z^{2}}-k_{\mathrm{r}}^{2} \alpha_{n}^{2}\right] E_{n}=B_{n} \sum_{p=1}^{N} E_{p}=B_{n} E}
\end{gathered}
$$

dans lesquelles :

et :

$$
\alpha_{n}=\frac{1-i \omega \tau}{l s_{n}}
$$

$$
B_{n}=\frac{3}{2} i \omega \mu_{0} \frac{\sigma}{l} \frac{1-s_{n}^{2}}{s_{n}} \alpha_{n} \Delta_{n}
$$

Pour des raisons de cohérence mathématique qui apparaîtront un peu plus loin lors de l'étude des conditions aux limites, chaque variable discrète doit être considérée comme la superposition de $N+1$ composantes soit :

$$
\begin{aligned}
& E_{n}=\sum_{p=1}^{N+1} a_{n p} \mathrm{e}^{i u_{p} z} \\
& J_{n}=\sum_{p=1}^{N+1} b_{n p} \mathrm{e}^{i u_{p} z} .
\end{aligned}
$$

Le champ électrique total sera donc de la forme :

$$
E(z)=\sum_{p=1}^{N+1} a_{p} \mathrm{e}^{i u_{p} z}
$$

Les égalités $(20 \mathrm{~b})$ peuvent donc s'écrire :

avec :

$$
\sum_{p=1}^{N+1} H_{n p} a_{n p} \mathrm{e}^{i u_{p} z}=B_{n} \sum_{m=1}^{N} \sum_{p=1}^{N+1} a_{m p} \mathrm{e}^{i u_{p} z}=B_{n} \sum_{p=1}^{N+1} a_{p} \mathrm{e}^{i u_{p} z}
$$

$$
H_{n p}=\left(u_{p}^{2}+\alpha_{n}^{2}\right)\left(u_{p}^{2}-k_{\mathrm{r}}^{2}\right) .
$$

L'identification des termes en $\mathrm{e}^{i u_{p} z}$ des $1^{\text {er }}$ et $3^{\mathrm{e}}$ membres de (22) donne la relation :

$$
a_{n p}=\frac{B_{n}}{H_{n p}} a_{p}, \quad n=1 \text { à } N \text {. }
$$


Les $2^{\mathrm{e}}$ et $3^{\mathrm{e}}$ membres de (22) impliquent la relation :

$$
a_{p}=\sum_{m=1}^{N} a_{m p}, \quad p=1 \text { à } \quad N+1 .
$$

Portant (23) dans (24), on obtient :

$$
\sum_{n=1}^{N} \frac{B_{n}}{H_{n p}} a_{p}=a_{p}
$$

soit encore :

$$
\sum_{n=1}^{N} \frac{B_{n}}{\left(u_{p}^{2}+\alpha_{n}^{2}\right)\left(u_{p}^{2}-k_{\mathrm{r}}^{2}\right)}=1 .
$$

Cette équation permet de calculer les $N+1$ vecteurs d'ondes $u_{p}$ car elle est équivalente à l'équation polynomiale de degré $N+1$ ci-dessous :

$$
\sum_{n} B_{n} \prod_{m \neq n}\left(X+\alpha_{m}^{2}\right)=\left(X-k_{\mathrm{r}}^{2}\right) \prod_{m}\left(X+\alpha_{m}^{2}\right) .
$$

La sommation et les produits sont effectués de 1 à $N$ et l'on a posé $X=u_{p}^{2}$.

L'amplitude des champs devant tendre vers 0 lorsque $z$ tend vers l'infini, les seules solutions physiquement acceptables sont celles qui satisfont à la condition :

$$
\operatorname{Im}\left\{u_{p}\right\}>0 \text {. }
$$

3.2 Conditions aUX Limites, AMPLitudes SPECTRALes. - Le problème sera entièrement résolu lorsqu'on aura déterminé les $N+1$ amplitudes $a_{p}$.

En effet, les $a_{n p}$ dépendent des $a_{p}$ par la relation (23) et les amplitudes $b_{n p}$ des composantes du courant sont, d'après l'équation (20 a) données par la relation :

$$
b_{n p}=\frac{u_{p}^{2}-k_{\mathrm{r}}^{2}}{i \omega \mu_{0}} a_{n p}
$$

soit encore :

$$
b_{n p}=\frac{B_{n}}{i \omega \mu_{0}} \frac{a_{p}}{u_{p}^{2}+\alpha_{n}^{2}} .
$$

L'expression du champ électrique total à l'interface $z=0$ donne une première équation :

$$
\sum_{p=1}^{N+1} a_{p}=E(z=0) \text {. }
$$

Les autres équations sont obtenues à partir des équations aux limites décrivant la façon dont les électrons sont diffusés par l'interface et exprimées par (16). Remplaçant dans cette dernière $j^{-}(z, s)$ par son expression (13b), on obtient :

$$
j(z=0, s)=\left(1+p_{0}\right) \frac{3}{4} \frac{\sigma}{l} \frac{1-s^{2}}{s} \int_{0}^{\infty} \mathrm{e}^{-\alpha t} E(t) \mathrm{d} t .
$$

Cela donne pour les variables discrètes :

$$
J_{n}(z=0)=\sum_{p=1}^{N+1} b_{n p}=\frac{1+p_{0}}{2 \alpha_{n}} \frac{B_{n}}{i \omega \mu_{0}} \int_{0}^{\infty} \mathrm{e}^{-\alpha_{n} t} \mathrm{~d} t \sum_{p=1}^{N+1} a_{p} \mathrm{e}^{i u_{p} t} .
$$

Utilisant l'expression (27) des $b_{n p}$, on obtient les $N$ équations supplémentaires nécessaires à la détermination $\operatorname{des} a_{p}$ :

$$
\sum_{p=1}^{N+1} \frac{\left(1-p_{0}\right) \alpha_{n}-\left(1+p_{0}\right) i u_{p}}{\alpha_{n}^{2}+u_{p}^{2}} a_{p}=0 \quad n=1 \text { à } N .
$$

Les $a_{p}$ sont donc solution du système de $N+1$ équations constitué de (28) et (29). 
Cela justifie le fait que dans le paragraphe précédent, on ait représenté chaque composante $E_{w} J_{n}$ par la somme de $N+1$ ondes.

On obtient finalement l'impédance de surface en appliquant la formule (1) :

$$
Z_{\mathrm{s}}=\omega \mu_{0} \frac{\sum_{p=1}^{N+1} a_{p}}{\sum_{p=1}^{N+1} a_{p} u_{p}}
$$

\section{Algorithmes de calcul.}

En résumé, le calcul de $Z_{\mathrm{s}}$ comporte les trois étapes suivantes :

- Recherche des $N+1$ vecteurs d'onde propres $u_{p}$ solutions de l'équation caractéristique (26).

- Recherche des $N+1$ amplitudes solutions du système d'équations $(28+29)$.

- Application de la formule (30).

On ne change rien au résultat si l'on suppose, dans (28), que $E(z=0)=1$.

L'impédance de surface s'écrira alors simplement :

$$
Z_{\mathrm{s}}=\frac{\omega \mu_{0}}{\sum_{p=1}^{N+1} a_{p} u_{p}} .
$$

Accessoirement, on peut calculer la variation spatiale du champ électrique :

$$
E(z)=\sum_{p=1}^{N+1} a_{p} \mathrm{e}^{i u_{p} z} .
$$

Il est à remarquer que les vecteurs donde $u_{p}$ ne dépendent pas des conditions aux limites; ces dernières n'interviennent que dans le calcul des amplitudes $a_{p}$.

\subsection{RÉSOlution DE l'éQuation CaRACtÉRISTIQUe. -} On écrira l'équation (26) sous la forme suivante :

$$
\begin{aligned}
C_{1} X^{n+1}+C_{2} X^{n}+ & C_{3} X^{n-1}+\cdots+ \\
& +C_{n+1} X+C_{n+2}=0 .
\end{aligned}
$$

Cela permettra d'appliquer les méthodes numériques standard. Ici $n=N$.

L'identification de (32) à (26) conduit aux formules de récurrence ci-dessous (voir annexe) :

$$
\begin{aligned}
C_{1} & =1 \\
C_{2} & =-Q_{0}+s_{1} \\
C_{3} & =-Q_{0} s_{1}+s_{2}-\mathcal{M}_{1} \\
-C_{p} & =-Q_{0} s_{p-2}+\delta_{p-1}-\mathfrak{L}_{p-3} \\
-C_{n+2} & =-Q_{0} \delta_{n}-\mathfrak{L}_{n-1} .
\end{aligned}
$$

On a posé $Q_{0}=k_{\mathrm{r}}^{2}$.
$S_{p}$ représente la somme de tous les produits possibles de $p$ termes parmi $N$ du type $\alpha_{i}^{2}$.

Cela peut s'écrire symboliquement :

$$
\begin{aligned}
& \left.\underset{\text { toutes les. }}{\delta_{p}=\sum^{\alpha_{i}^{2} \alpha_{j}^{2}----\alpha_{k}^{2}}}\right) . \\
& \text { combinaisons de } p \text { termes }
\end{aligned}
$$

On a comme précédemment :

$$
\alpha_{i}=\frac{1-i \omega \tau}{l s_{i}}
$$

$\mathcal{M}_{p}$ est définie par la relation :

$$
\mathcal{M}_{p}=(-1)^{p-1} \sum_{m=1}^{n} B_{m} \alpha_{m}^{2(p-1)}
$$

avec :

$$
B_{m}=\frac{3}{2} i \omega \mu_{0} \frac{\sigma}{l} \frac{1-\mathrm{s}_{m}^{2}}{s_{m}} \alpha_{m} \Delta_{m}
$$

$\mathfrak{L}_{p}$ est définie par la série :

$$
\mathcal{L}_{p}=S_{p} \mathcal{M}_{1}+S_{p-1} \mathcal{M}_{2}+\cdots+S_{1} \mathcal{M}_{p}+\mathcal{M}_{p+1}
$$

D'un point de vue pratique, des difficultés peuvent apparaître lors du calcul de (35) dans laquelle figurent des termes $\alpha_{m}^{2(p-1)}$; la valeur maximale de l'exposant est $2(N-1)$ et si $N$ est grand, un dépassement de la capacité de l'ordinateur à représenter des nombres très grands ou très petits peut se produire.

Les résultats numériques présentés au $\S 5$ ont été obtenus au moyen d'un micro-ordinateur LSI-11 dont le compilateur FORTRAN ne permet pas de traiter des variables qui soient à la fois complexes et représentées par des nombres en double précision.

En conséquence, il ne nous a pas été possible de dépasser $N=6$ en dépit des précautions prises dans l'établissement des programmes.

Cette restriction n'est cependant pas très gênante dans la mesure où il suffit de prendre $N$ égal à 4 ou 5 pour atteindre les précisions habituellement requises.

Nous avons essayé deux méthodes itératives similaires pour résoudre l'équation polynomiale (32) :

- celle de Bairstow ([11] page 747).

- celle de Birge Vieta [12].

C'est cette dernière qui s'est avérée être la plus fiable quant à la convergence des calculs. 
Si $N$ est trop grand (supérieur à 10 environ), la convergence devient incertaine et le recours à une méthode plus sûre bien que plus délicate à mettre en ouvre telle que celle de Bernouilli ([13] page 387) est à envisager.

4.2 Calcul NumériQue Des amplitudes. - Les amplitudes spectrales $a_{p}$ sont solutions du système $(28+29)$ dans lequel nous avons fait $E(z=0)=1$.

Nous avons utilisé une méthode classique de transformation « par pivots " ([13] page 72).

La matrice représentative du système est toujours régulière et le calcul ne nécessite aucune précaution particulière.

4. 3 EQUATIONS SIMPLIFIÉES OBTENUES POUR $N=1$. Si l'on fait $N=1$, l'équation caractéristique (26) se réduit à :

avec :

$$
X^{2}+\alpha^{2} X-B=0
$$

$$
\alpha=\frac{1-i \omega \tau}{l s}
$$

et :

$$
B=\frac{3}{2} i \omega \mu_{0} \frac{\sigma}{l} \frac{1-s^{2}}{s} \alpha \Delta s .
$$

On a négligé le courant de déplacement c'est-à-dire le terme $k_{\mathrm{r}}$. Les racines en sont :

$$
\begin{aligned}
& X_{1}=\frac{\alpha^{2}}{2}(-1+\sqrt{1+\beta}) \\
& X_{2}=\frac{\alpha^{2}}{2}(-1-\sqrt{1+\beta}) .
\end{aligned}
$$

On a posé :

$$
\beta=\frac{4 B}{\alpha^{4}}
$$

Les vecteurs d'onde propres seront donc :

$$
u_{i}=X_{i}^{1 / 2} \text { avec } \operatorname{Im}\left\{u_{i}\right\}>0 .
$$

C'est-à-dire que l'on considère le champ électrique comme la superposition de deux ondes :

$$
E(z)=a_{1} \mathrm{e}^{i u_{1} z}+a_{2} \mathrm{e}^{i u_{2} z} .
$$

Les équations (28) et (29) se réduisent à :

$$
a_{1}+a_{2}=1
$$

$$
\begin{aligned}
& \frac{\left(1-p_{0}\right) \alpha-\left(1+p_{0}\right) i u_{1}}{u_{1}^{2}+\alpha^{2}} a_{1}+ \\
& +\frac{\left(1-p_{0}\right) \alpha-\left(1+p_{0}\right) i u_{2}}{u_{2}^{2}+\alpha^{2}} a_{2}=0 .
\end{aligned}
$$

L'impédance de surface s'écrira simplement :

$$
Z_{\mathrm{s}}=\frac{\omega \mu_{0}}{a_{1} u_{1}+a_{2} u_{2}} .
$$

Afin de tester la validité de cette méthode de calcul, il est intéressant de chercher l'expression approchée de $Z_{\mathrm{s}}$ aux longueurs d'onde de l'ordre du $\mu \mathrm{m}$.

On peut alors considérer comme vérifiées les deux inégalités $\omega \tau \gg 1$ et $|\beta| \ll 1$.

Par exemple, pour le cuivre à température ambiante et $\lambda=1 \mu \mathrm{m}$, on obtient :

$$
\begin{aligned}
& \omega \tau \approx 40 \\
& |\beta| \approx 1,3 \times 10^{-3} .
\end{aligned}
$$

Les vecteurs d'onde précédents s'écriront :

$$
\begin{aligned}
& u_{1} \#-\frac{\alpha}{2} \beta^{1 / 2} \\
& u_{2} \# i \alpha .
\end{aligned}
$$

La résolution de (37) donne l'expression suivante de l'impédance de surface :

$$
Z_{\mathrm{s}}=\omega \mu_{0} \frac{\left(1-p_{0}\right)\left(u_{1}+u_{2}\right) \alpha-\left(1+p_{0}\right) i u_{1} u_{2}+\left(1+p_{0}\right) i \alpha^{2}}{\left(1-p_{0}\right)\left[\left(u_{1}-u_{2}\right)^{2}+\alpha^{2}+3 u_{1} u_{2}\right] \alpha-\left(1+p_{0}\right) i u_{1} u_{2}\left(u_{1}+u_{2}\right)}
$$

Considérant comme négligeables devant l'unité les puissances de $\beta$ supérieures à $1 / 2$, on obtient finalement :

$$
Z_{\mathrm{s}} \#-\frac{2 \omega \mu_{0}}{\beta^{1 / 2} \alpha}\left[1+\left(1-p_{0}\right) \frac{i}{4} \beta^{1 / 2}\right] .
$$

Dans les expressions de $\beta$ et $\alpha$ interviennent les quantités $s$ et $\Delta s$.

Leurs valeurs ne sont pas quelconques si l'on impose les conditions suivantes :

- d'une part, pour une faible anomalie et $p=1$, le courant de conduction doit se raccorder à son expression classique (8) (voir le paragraphe « Erreur de troncature " en Annexe);

- d'autre part, la composante $v_{z}$ de la vitesse des électrons de conduction est égale à sa valeur moyenne.

On est alors conduit aux relations ci-dessous :

$$
s=\frac{\left\langle v_{z}\right\rangle}{v_{\mathrm{F}}}=\frac{1}{v_{\mathrm{F}}} \frac{\iiint v_{z} f_{0}(v) \mathrm{d}^{3} v}{\iiint f_{0}(v) \mathrm{d}^{3} v}=\frac{3}{8}
$$

et $\left(1-s^{2}\right) \Delta s=2 / 3$. 
Les expressions approchées de $\alpha$ et $\beta^{1 / 2}$ sont donc :

$$
\begin{gathered}
\alpha \#-\frac{8}{3} i \frac{\omega \tau}{l}\left(1+\frac{i}{\omega \tau}\right) \\
\beta^{1 / 2} \# \frac{3}{4} \frac{l \sqrt{\sigma \omega \mu_{0}}}{(\omega \tau)^{3 / 2}}\left(1-\frac{3}{2} \frac{i}{\omega \tau}\right) .
\end{gathered}
$$

Portant ces expressions dans (38) et utilisant les relations $\sigma=\omega_{p}^{2} \tau \varepsilon_{0}$ et $Z_{0}=c \mu_{0}$, on obtient :

$$
Z_{\mathrm{s}}=Z_{0}\left\{\frac{1}{2 \omega_{p} \tau}\left[1+\left(1-p_{0}\right) \frac{3}{8} k_{p} l\right]-i \frac{\omega}{\omega_{p}}\right\}
$$

avec $: k_{p}=\omega_{p} / c$.

Pour les valeurs limites 1 et 0 de $p_{0}$, on retrouve les expressions données respectivement par la théorie classique et par les deux premiers termes du développement en série de Dingle [3] dans les mêmes conditions d'approximation.

Le point important de ce calcul est la confirmation de l'existence du facteur $\left(1-p_{0}\right)$ dans la partie réelle de $Z_{\mathrm{s}}$.

\section{Résultats numériques.}

Les calculs ont été effectués pour le cuivre caractérisé par les deux constantes :

$$
\begin{aligned}
& \frac{\sigma}{l}=1,515 \times 10^{15} \Omega^{-1} \mathrm{~m}^{-2} \\
& v_{\mathrm{F}}=1,59 \times 10^{6} \mathrm{~ms}^{-1}
\end{aligned}
$$

L'impédance de surface sera notée sous forme complexe :

ici $X<0$.

$$
Z_{\mathrm{s}}=R+i X
$$

On désignera par $R_{\mathrm{cl}}$ et $X_{\mathrm{cl}}$ les parties réelles et imaginaires calculées au moyen de la théorie locale classique.

\section{1 Précision de la mÉTHOde. - La comparaison des} résultats obtenus par cette méthode à ceux donnés par les séries de Dingle pour $p=0$ et 1 (Figs. 3 à 6) montre qu'aux longueurs d'onde inférieures à $200 \mu \mathrm{m}$ environ, la valeur $N=4$ donne une précision de l'ordre de 2 à $3 \%$.

A titre indicatif, les résultats numériques obtenus à $\lambda=10 \mu \mathrm{m}$ sont portés dans le tableau I.

On a discrétisé la variable $s$ en découpant simplement son domaine $(0,1)$ de variation en intervalles $\Delta_{1}, \Delta_{2}, \ldots, \Delta_{N}$ égaux et en choisissant les échantillons $s_{1}, s_{2}, \ldots, s_{N}$ au centre des intervalles correspondants.

La part de l'erreur due, dans ces conditions, aux effets de troncature est de l'ordre de $1 \%$ pour $N=4$ (voir annexe) et explique le léger écart apparaissant

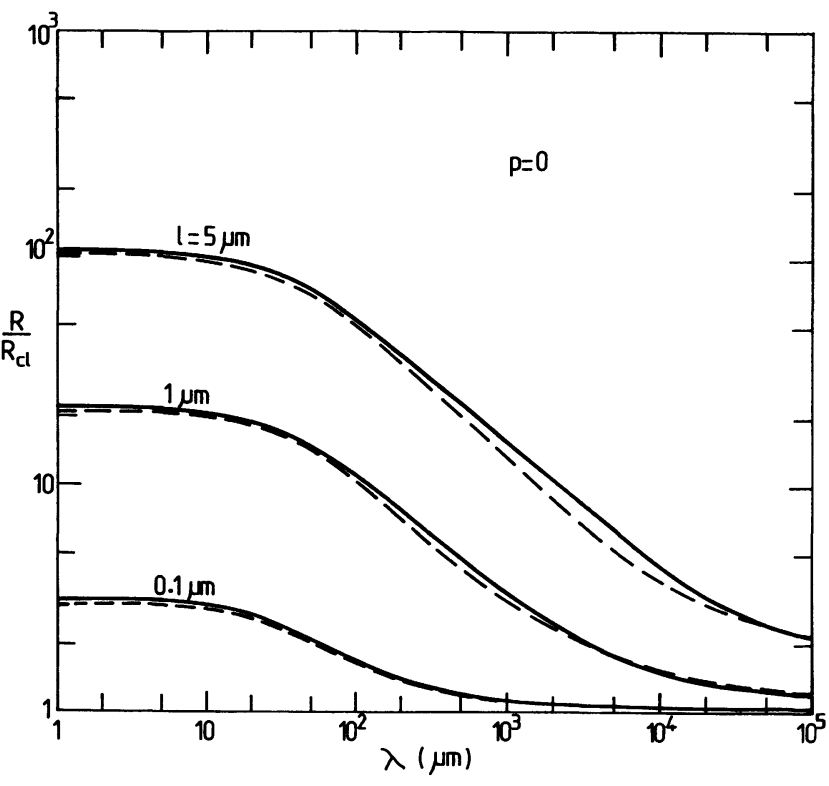

Fig. 3. - Résistance de surface de cuivre rapportée à sa valeur classique pour la réflexion complètement diffuse $(p=0)$ des électrons. courbes calculées par notre méthode $(N=4)$. - . - . courbes calculées au moyen des séries de Dingle.

[Surface resistance of Copper, reported to its classical value in the case of diffuse scattering $(p=0)$ of the electrons. our results $(N=4) \ldots .$. results obtained from Dingle series.]

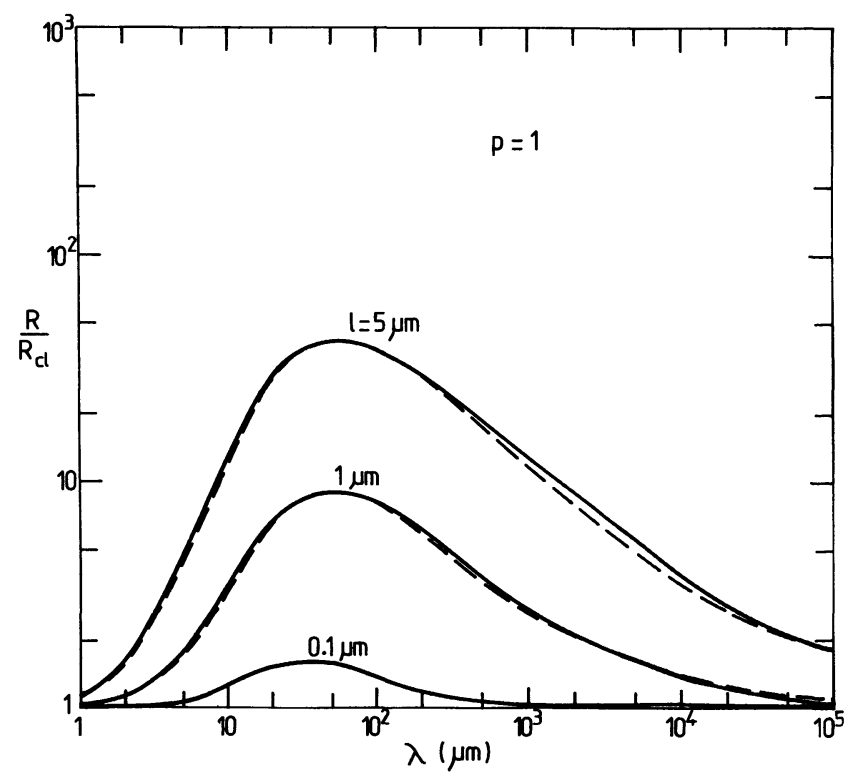

Fig. 4. - Résistance de surface de cuivre rapportée à sa valeur classique pour la réflexion complètement spéculaire $(p=1)$ des électrons. courbes calculées par notre méthode $(N=4)$. . . . - courbes calculées au moyen des séries de Dingle.

[Surface resistance of Copper, reported to its classical value in the case of specular reflexion $(p=1)$ of the electrons. our results $(N=4)$. - . - - results obtained from Dingle series.] 


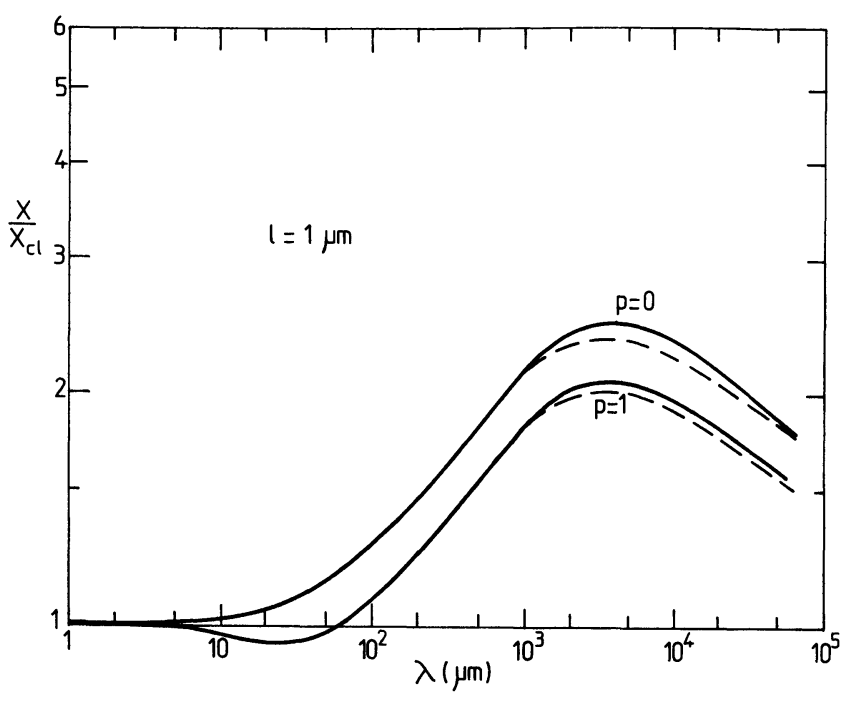

Fig. 5. - Réactance de surface du cuivre rapportée à sa valeur classique pour un libre parcours moyen $l=1 \mu \mathrm{m}$. - courbes calculées par notre méthode $(N=4)$. - . - - courbes calculées au moyen des séries de Dingle.

[Surface reactance of Copper, reported to its classical value for a $\operatorname{lpm} l=1 \mu \mathrm{m}, \longrightarrow$ our results $(N=4)$. . . . . results obtained from Dingle series.]

aux courtes longueurs d'onde sur les courbes de la figure 3.

Les courbes de la figure 7 montrent que la valeur minimale $N=1$ conduit à des résultats très satisfaisants aux longueurs d'onde inférieures à $10 \mu \mathrm{m}$ lorsque la réflexion des électrons est essentiellement diffuse.

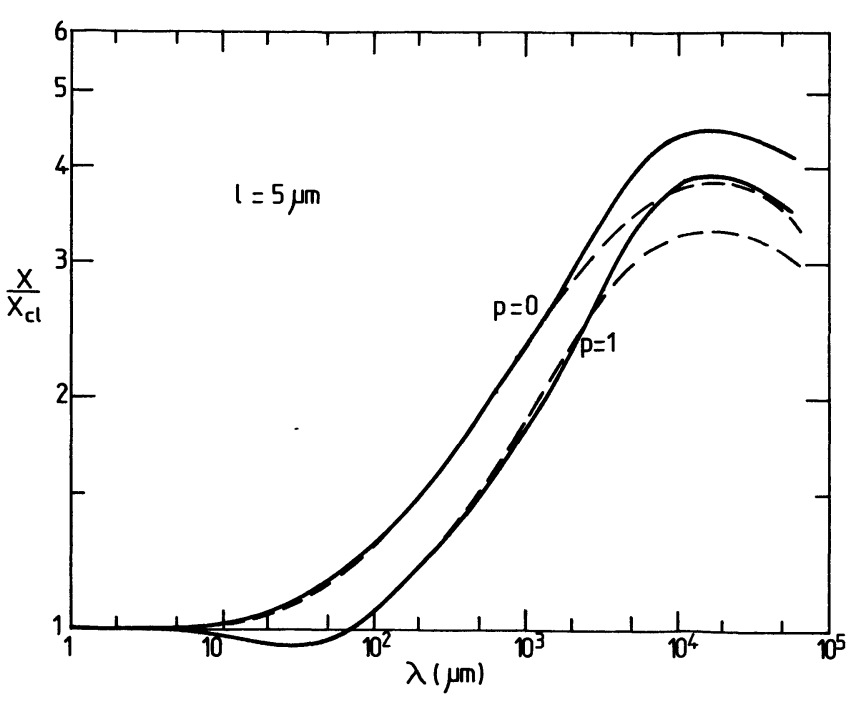

Fig. 6. - Réactance de surface du cuivre rapportée à sa valeur classique pour un libre parcours moyen $l=5 \mu \mathrm{m}$. - courbes calculées par notre méthode $(N=4)$. . . . - courbes calculées au moyen des séries de Dingle.

[Same as figure 5, for $l=5 \mu \mathrm{m}$.]

L'erreur de troncature a été minimisée du fait que l'on a donné à $s$ et à $\Delta s$ les valeurs satisfaisant aux conditions mentionnées au $\S 4.3$ précédent.

Cela explique que courbes exactes et approchées coïncident rigoureusement pour $p=0$.

Le principal intérêt des algorithmes définis au chapitre 4 est de permettre le calcul de $Z_{\mathrm{s}}$ pour $p$ quelconque.

Tableau I. - Comparaison des résultats obtenus par les séries de Dingle et par notre méthode à $\lambda=10 \mu \mathrm{m}$ pour $N=4$. l est le libre parcours moyen. Dans chaque case on a porté les rapports $R / R_{\mathrm{cl}}$ (partie supérieure) et $X / X_{\mathrm{cl}}$ (partie inférieure).

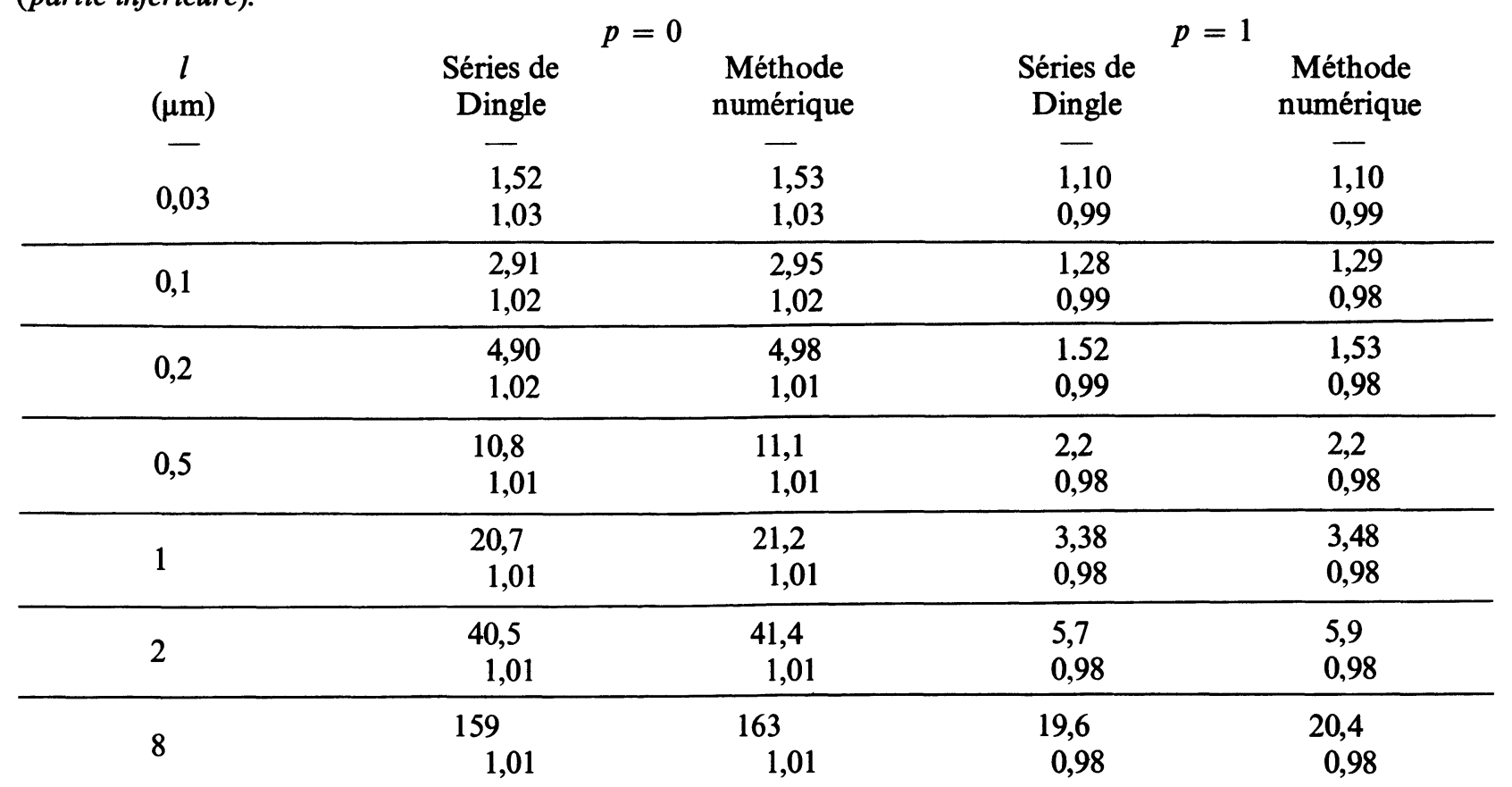




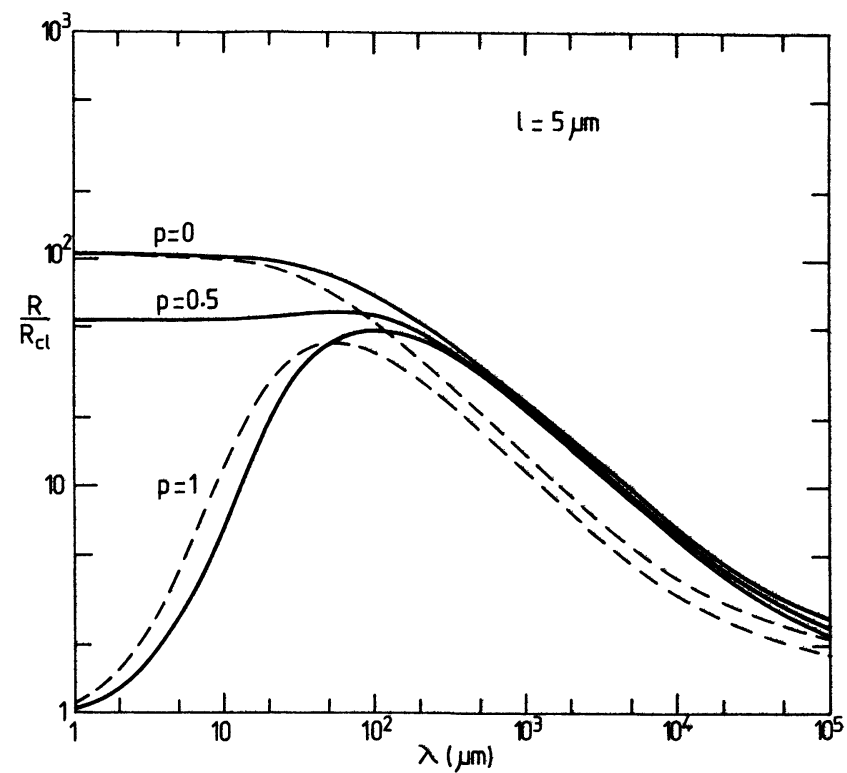

Fig. 7. - Résistance de surface calculée par notre méthode pour $N=1$ et $l=5 \mu \mathrm{m}$ (trait continu). La courbe obtenue pour $p=0$ se raccorde exactement à la courbe calculée au moyen des séries de Dingle (trait interrompu) aux courtes longueurs d'onde, à cause des valeurs particulières données aux quantités $s$ et $\Delta s$ (voir $\S 4.3$ ).

[Surface resistance of copper obtained with $N=1$ and $l=5 \mu \mathrm{m}$ (continuous line). The curve corresponding to $p=0$ fit exactly the curve obtained from the Dingle series at short wavelengths.]

On a représenté sur la figure 8 les courbes obtenues pour $p=0,5$. On peut raisonnablement supposer que, toutes choses égales par ailleurs, la précision des calculs dépend peu de la valeur de $p$.

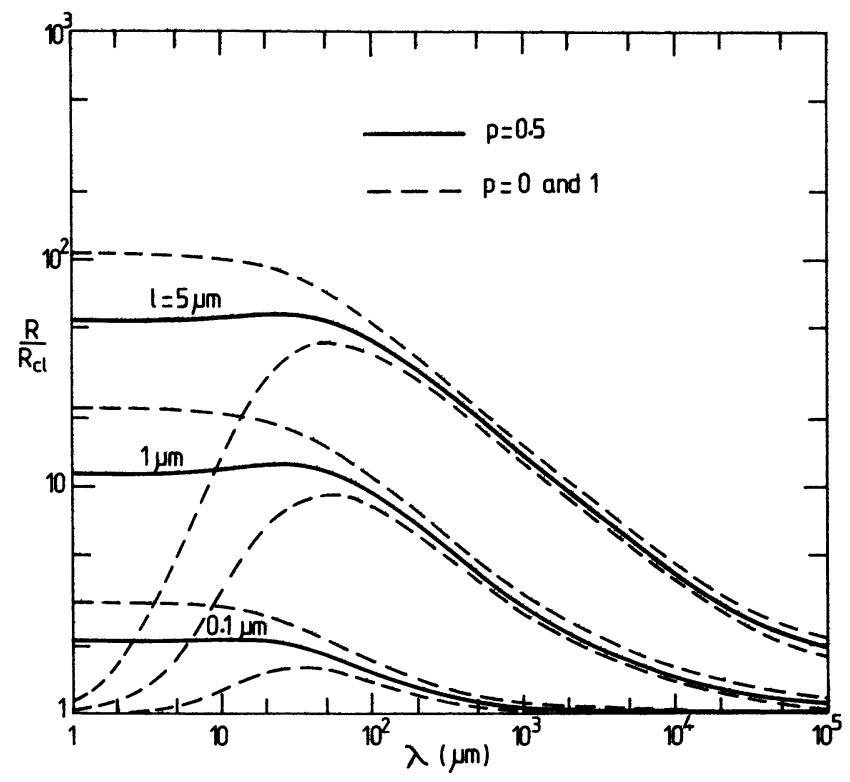

Fig. 8. - Résistance de surface du cuivre obtenue par notre méthode pour $p=0,5$ (trait continu).

[Surface resistance of copper for $p=0.5$ (continuous line).] REVUE DE PHYSIQUE APPLßÚÉE. - T. 19, No 8, AOOT 1984
5.2 INFLUENCE DU PARAMÈTRE DE DIFFUSION. - On a représenté sur la figure 9 la partie réelle de l'impédance de surface pour différentes valeurs de $p$.

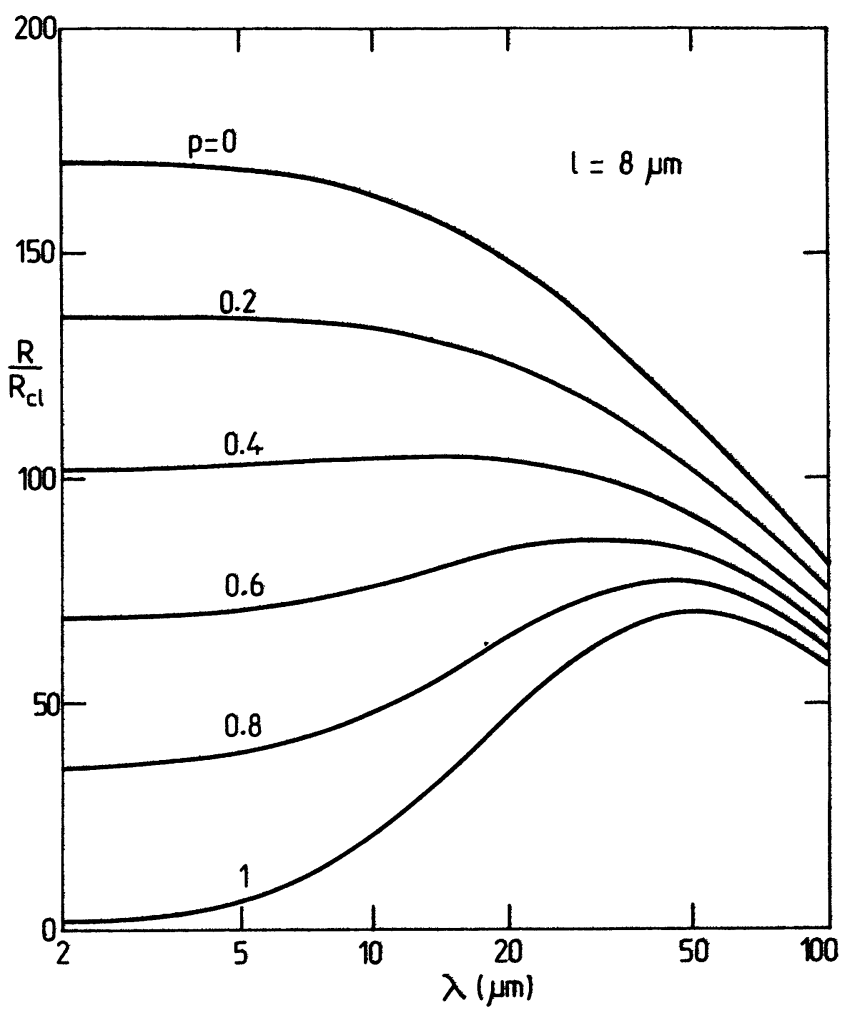

Fig. 9. - Résistance de surface obtenue pour différentes valeurs du paramètre $p$ de diffusion des électrons dans le cas d'une forte anomalie $(l=8 \mu \mathrm{m})$. Le calcul a été effectué avec $N=4$. L'échelle verticale est linéaire.

[Surface resistance for different values of $p$ in the region of an important anomalous skin effect $(\lambda=8 \mu \mathrm{m})$ with $N=4$. Vertical scale is linear.]

L'échelle verticale étant linéaire, l'espacement régulier entre courbes à $\lambda$ donné suggère d'écrire la partie réelle de limpédance de surface sous la forme :

$$
R=p R_{1}+(1-p) R_{0}
$$

$R_{0}$ et $R_{1}$ étant les valeurs calculées respectivement pour $p=0$ et 1 .

L'écart en pourcentage du résultat de (40) par rapport à la valeur exacte $R_{\mathrm{ex}}$ est défini par :

$$
\delta(p)=100 X \frac{R-R_{\mathrm{ex}}}{R_{\mathrm{ex}}} .
$$

Chaque point de la figure 10 est la moyenne arithmétique $\langle\delta\rangle_{p}$ des écarts obtenus dans le domaine de variation $(0,1)$ de $p$.

Le réseau de courbes ainsi tracé montre que l'expression approchée (40) est remarquablement bien vérifiée aux courtes longueurs d'ondes $(\lambda<5 \mu \mathrm{m})$; elle demeure satisfaisante $\left(\langle\delta\rangle_{p}<4 \%\right)$ aux longueurs 


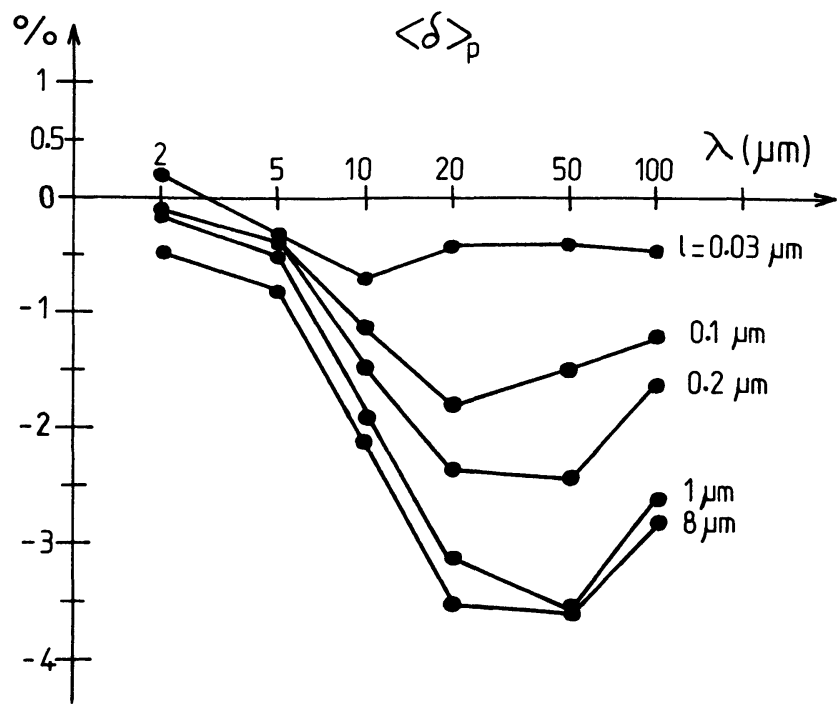

Fig. 10. - Valeur moyenne de l'écart entre la résistance de surface calculée au moyen de la formule approchée (40) et la valeur supposée exacte calculée par notre méthode. A une longueur d'onde $\lambda$ et un libre parcours moyen $l$ donnés, cet écart moyen est défini par :

$$
\langle\delta\rangle_{p}=\frac{1}{m} \sum_{i=1}^{m} \delta\left(p_{i}\right)
$$

$\delta\left(p_{i}\right)$ est défini dans le $\S 5.2$ et on a pris ici 6 valeurs $p_{i}$ régulièrement espacées entre 0 et 1 .

[Mean deviations between surface resistance values calculated by using either the simplified formula (40) or our method. At given values of the wavelength $\lambda$ and $1 \mathrm{pm} l$ respectively, the mean deviation is defined by :

$$
\left\langle\delta_{p}\right\rangle=\frac{1}{m} \sum_{i=1}^{m} \delta\left(p_{i}\right)
$$

$\delta\left(p_{i}\right)$ is given in 5.2 , and 6 values of $p_{i}$ between 0 and 1 are used.]

d'onde plus grandes lorsque l'anomalie n'est pas trop importante.

Aux longueurs d'onde supérieures à $100 \mu \mathrm{m}$ environ, ces considérations perdent de leur intérêt car les courbes $R_{0}(\lambda)$ et $R_{1}(\lambda)$ tendent à se confondre.

Dans une précédente publication [16], nous avions calculé la distance d'amortissement $L$ d'une onde de plasma de surface (OPS) se propageant à l'interface cuivre-vide dans le domaine $2<\lambda<10 \mu \mathrm{m}$ et proposé la relation empirique :

$$
\mathcal{L}(\sigma) \equiv \frac{L_{\mathrm{c} 1}}{L}-1 \# m \sigma
$$

dans laquelle $m$ est donné par :

$$
m=p m_{1}+(1-p) m_{0}
$$

$L_{\mathrm{cl}}$ est la distance d'amortissement calculée par la théorie locale classique.
$L$ est la distance d'amortissement exacte. Elle est égale, par définition, à $1 / \mathfrak{J m}\left\{k_{x}\right\}$, le vecteur d'onde tangentiel $k_{x}$ de l'OPS étant donné par la relation de dispersion [16] :

$$
k_{x}=\frac{\omega}{c}\left[1-\left(\frac{Z_{\mathrm{s}}}{Z_{0}}\right)^{2}\right]^{1 / 2}
$$

$\sigma$ est la conductivité du métal massif en courant continu. Si (41) est exacte, la fonction $\mathcal{L}(\sigma)$ doit donc être représentée, à $\lambda$ donnée, et $p$ étant choisi comme paramètre, par un réseau de droites dont les pentes extrêmes $m_{0}$ et $m_{1}$ correspondent à $p=0$ et 1 respectivement.

Le calcul effectué pour $N=4$ et $\lambda=10 \mu \mathrm{m}$ donne effectivement un réseau de droites, représenté sur la figure 11 , dont les pentes diffèrent au maximum de $5 \%$ de l'expression approchée (41).

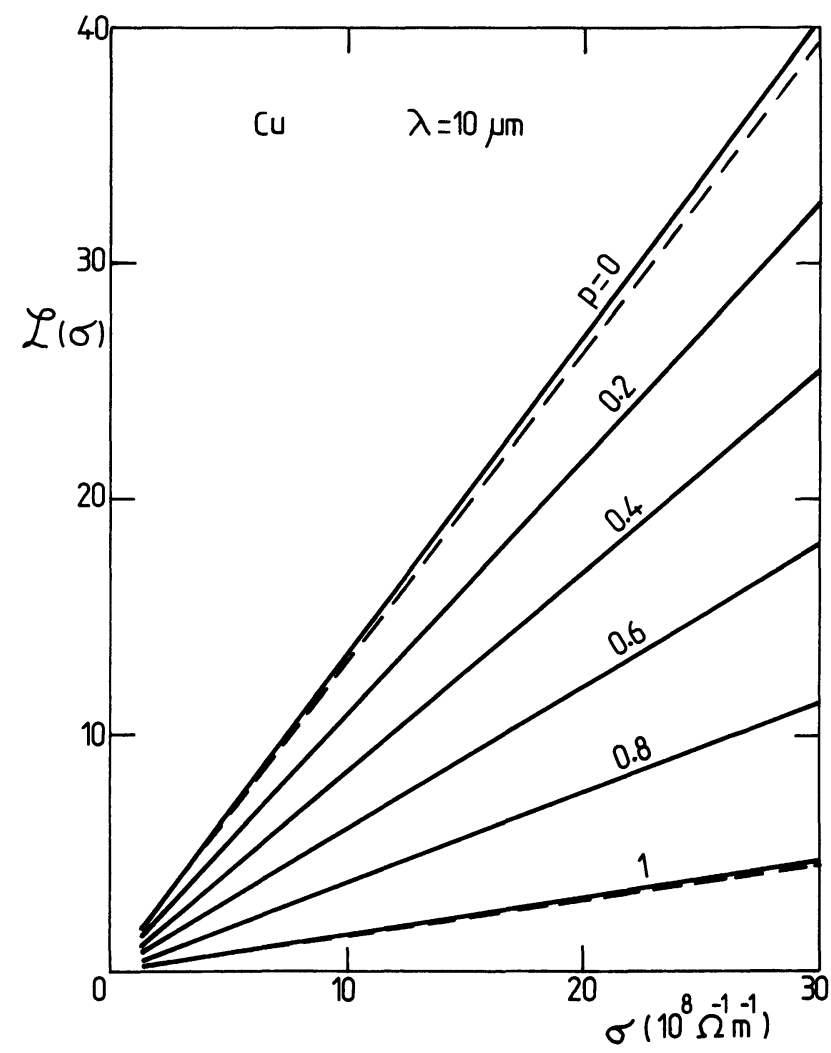

Fig. 11. - Réseau de courbes $\mathfrak{\complement}(\sigma)$ (voir la définition au $§ 5.2$ ) paramétré en $p$ (trait continu). On a tracé en trait interrompu les droites extrêmes obtenues pour $p=0$ et 1 au moyen des séries de Dingle.

[Curves giving $\mathfrak{L}(\sigma)^{\circ}$ for different values of $p$ (continuous lines), and straight lines (dotted lines) obtained at $p=0$ and 1 respectively from the Dingle series.]

Compte tenu des bons résultats obtenus par notre méthode de calcul aux courtes longueurs d'onde, on peut estimer que cet écart de $5 \%$ constitue la précision pouvant être espérée de (41).

Bien qu'il n'existe pas, en général, de relation simple 
entre (40) et (41), il est possible, aux longueurs d'onde suffisamment courtes $(\lambda<5 \mu \mathrm{m})$ de les déduire de l'expression approchée (39) du fait que l'on peut écrire :

$$
\begin{array}{ll} 
& R_{1} \# R_{\mathrm{cl}} \\
\text { et } \quad & X \# X_{\mathrm{cl}} \text { pour tout } p
\end{array}
$$

ce qui implique $m_{1} \approx 0$.

\section{Cas d'une diffusion anisotrope.}

On considère le cas d'un paramètre de diffusion dépendant de l'angle d'incidence des électrons sur l'interface, c'est-à-dire de $s$; on ne s'intéressera, en outre, qu'aux longueurs d'onde permettant d'exprimer la résistance de surface par la relation approchée (40).

Si $p$ est une fonction de $s$, on doit pouvoir écrire (40) sous la forme :

$$
R=\langle p\rangle R_{1}+(1-\langle p\rangle) R_{0}
$$

avec :

$$
\langle p\rangle=\int_{0}^{1} p(s) w(s) \mathrm{d} s
$$

$w(s)$ est une fonction de pondération à déterminer telle que :

$$
\int_{0}^{1} w(s) \mathrm{d} s=1
$$

Pour les variables discrètes, on écrira :

$$
\langle p\rangle=\sum_{i=1}^{N} p_{i} W_{i}
$$

avec :

$$
W_{i}=\int_{s_{i}-\frac{\Delta s}{2}}^{s_{i}+\frac{\Delta s}{2}} w(s) \mathrm{d} s
$$

et :

$$
\sum_{i=1}^{N} W_{i}=1
$$

Un moyen de déterminer chaque $W_{i}$ est de calculer la résistance de surface $R_{i}$ correspondant à un paramètre de diffusion défini par la distribution $p_{j}=\delta_{i j}$. $\delta_{i j}$ est le symbole de Kronecker.

On obtient alors à partir de (42) :

$$
W_{i}=\frac{R_{0}-R_{i}}{R_{0}-R_{1}}
$$

Il est ensuite aisé, connaissant $R_{0}, R_{1}$ et les $W_{i}$, de calculer $R$ pour une distribution $p_{j}$ quelconque, au moyen des formules (42) et (43).

On a porté dans le tableau II les coefficients de pondération obtenus pour $N=4$. Ils varient peu dans le domaine $2<\lambda<10 \mu \mathrm{m}$.

L'obtention du maximum pour $i=3$, c'est-à-dire pour $0,50<s<0,75$ montre que ce sont les électrons ayant un angle d'incidence compris entre environ 40
Tableau II. - Valeurs des coefficients $W_{i}$ pour $N=4$ aux longueurs d'onde 2,5 et $10 \mu \mathrm{m}$. Le libre parcours moyen l vaut $5 \mu \mathrm{m}$.

\begin{tabular}{|c|c|c|c|c|}
\hline$\lambda_{(\mu \mathrm{m})}^{i}$ & 1 & 2 & 3 & 4 \\
\hline 2 & 0,121 & 0,314 & 0,369 & 0,197 \\
\hline 5 & 0,126 & 0,325 & 0,371 & 0,190 \\
\hline 10 & 0,145 & 0,357 & 0,371 & 0,167 \\
\hline
\end{tabular}

et $60^{\circ}$ qui sont les plus « sensibles » aux conditions de diffusion par la surface.

On a donné dans le tableau III les valeurs de $R$ obtenues pour différentes distributions $p_{j}$.

L'accord entre le calcul exact et le calcul simplifié est excellent.

Tableau III. - Comparaison des valeurs exactes et approchées de $R$ pour différentes distributions $p_{j} d u$ paramètre de diffusion. Les données $d u$ calcul sont : $N=4, \quad \lambda=5 \mu \mathrm{m}, \quad l=1 \mu \mathrm{m}, \quad R_{0} / R_{\mathrm{cl}}=21,85$, $R_{1} / R_{\mathrm{cl}}=1,75$.

Les $W_{i}$ sont de $i=1 \grave{a} 4: 0,126-0,325-0,371-0,190$.

\begin{tabular}{|c|l|l|l|c|c|}
\hline \multicolumn{5}{|c|}{$P_{j}, j=$} & \multicolumn{2}{c|}{$R / R_{\mathrm{cl}}$} \\
\hline 1 & 2 & 3 & 4 &. Exacte & Approchée \\
\hline 1 & 0,3 & 0,1 & 0 & 16,61 & 16,60 \\
\hline 0,2 & 0,5 & 0,2 & 0,2 & 15,82 & 15,82 \\
\hline 0,5 & 0,1 & 0,1 & 0,2 & 18,42 & 18,41 \\
\hline 1 & 0 & 1 & 0 & 11,88 & 11,86 \\
\hline 1 & 1 & 1 & 1 & 5,43 & 5,33 \\
\hline
\end{tabular}

5.3 VARIATION SPATIALE DES CHAMPS ET DES DENSITÉS DE COURANT. - La variation spatiale du champ électrique est représentée sur la figure 12 pour différentes longueurs d'onde dans le cas d'une forte anomalie $(l=5 \mu \mathrm{m}, p=0)$. L'échelle des distances est rapportée à la profondeur de pénétration classique $1 / \mathrm{Jm}\left[k_{\mathrm{m}}\right]$ de l'amplitude du champ, $k_{\mathrm{m}}$ désignant le vecteur d'onde dans le métal.

Dans le cadre de la théorie classique, toutes les courbes devraient être confondues avec l'exponentielle $\mathrm{e}^{-\boldsymbol{x}}$. En dépit de la forte anomalie, cela est pratiquement vrai pour $\lambda=10 \mu \mathrm{m}$. Au-delà de cette valeur, 




Fig. 12. - Variation spatiale de l'amplitude du champ électrique dans le métal. On a porté en abscisses $(x)$ la cote $z$ dans le métal (Fig. 1) rapportée à la profondeur de pénétration classique $1 / J_{\mathrm{m}}\left\{k_{\mathrm{m}}\right\}, k_{\mathrm{m}}$ désignant le vecteur d'onde de Drude.

[Spatial variations of the electriec field in the metal as a function of the distance from the surface $z$ reported to the classical penetration depth $\left[\mathfrak{J}_{\mathrm{m}}\left(k_{\mathrm{m}}\right)\right]^{-1}, k_{\mathrm{m}}$ being the Drude wave vector.]

la profondeur de pénétration rapportée à sa valeur classique croît avec la longueur d'onde.

Dans le proche infrarouge, la loi de variation de l'amplitude du champ électrique ne peut être distinguée de l'exponentielle classique.

Il est donc légitime d'attribuer à l'onde dans le métal un vecteur d'onde unique $k_{\mathrm{m}}$ lié aux paramètres de Drude $\omega_{\mathrm{p}}$ et $\tau^{*}$ par la relation :

$$
k_{\mathrm{m}} \# \frac{\omega_{\mathrm{p}}}{c}\left(\frac{1}{2 \omega \tau^{*}}+i\right)
$$

valable si $\omega \tau^{*} \gg 1$ et $\left(\omega / \omega_{\mathrm{p}}\right)^{2} \ll 1$ :

La formule (1) de définition de l'impédance de surface permet d'écrire :

$$
k_{\mathrm{m}}=\frac{\omega \mu_{0}}{Z_{\mathrm{s}}}
$$

Soit, d'après (39) :

$$
k_{\mathrm{m}} \# \frac{\omega_{\mathrm{p}}}{c}\left\{\frac{1}{2 \omega \tau}\left[1+(1-p) \frac{3}{8} k_{\mathrm{p}} l\right]+i\right\} .
$$

On trouve donc bien $[9,10]$ que la diffusion des électrons par la surface a pour effet de diminuer le temps de relaxation intervenant dans la constante diélectrique " apparente » de Drude du métal.

L'identification des deux expressions de $k_{\mathrm{m}}$ donne :

$$
\frac{1}{\tau^{*}}=\frac{1}{\tau}\left[1+(1-p) \frac{3}{8} k_{p} l\right] \text {. }
$$

Par ailleurs, on remarque sur la figure 12 que la courbe obtenue pour $\lambda=100 \mu \mathrm{m}$ présente une oscilla- tion assez marquée par rapport à son allure probable, au-delà de $x \simeq 1,5$ soit $z \simeq 300 \AA$.

Cela provient de ce que le champ calculé ne se confond rigoureusement avec le champ exact que pour $z \rightarrow 0$. Comme d'après la formule (1), c'est le gradient du champ en $z=0^{+}$qui détermine la valeur de $Z_{\mathrm{s}}$, ce phénomène ne nuit guère à la précision du calcul dans le cas d'un métal semi-infini.

Il n'en serait pas de même dans le cas d'une couche mince d'épaisseur $d$ pour laquelle il faudrait inclure dans les conditions aux limites le gradient du champ en $z=d^{-}$, des oscillations des courbes $Z_{\mathrm{s}}(d)$ étant alors à craindre si $N$ nest pas suffisamment grand.

La figure 13 représente le module de l'amplitude des courants $J_{n}$ définis par les expressions $(21 \mathrm{~b})$ et (27), pour $N=5$. Chacun d'eux est la superposition de termes en $\exp ( \pm \alpha z)$ avec :

$$
\alpha=\frac{1-i \omega \tau}{l s} .
$$

Les valeurs de s associées à $J_{1}, J_{2}, \ldots, J_{5}$ sont respectivement de l'ordre de $0,1,0,3, \ldots, 0,9$, et expliquent la dépendance de la période des oscillations avec $n$.

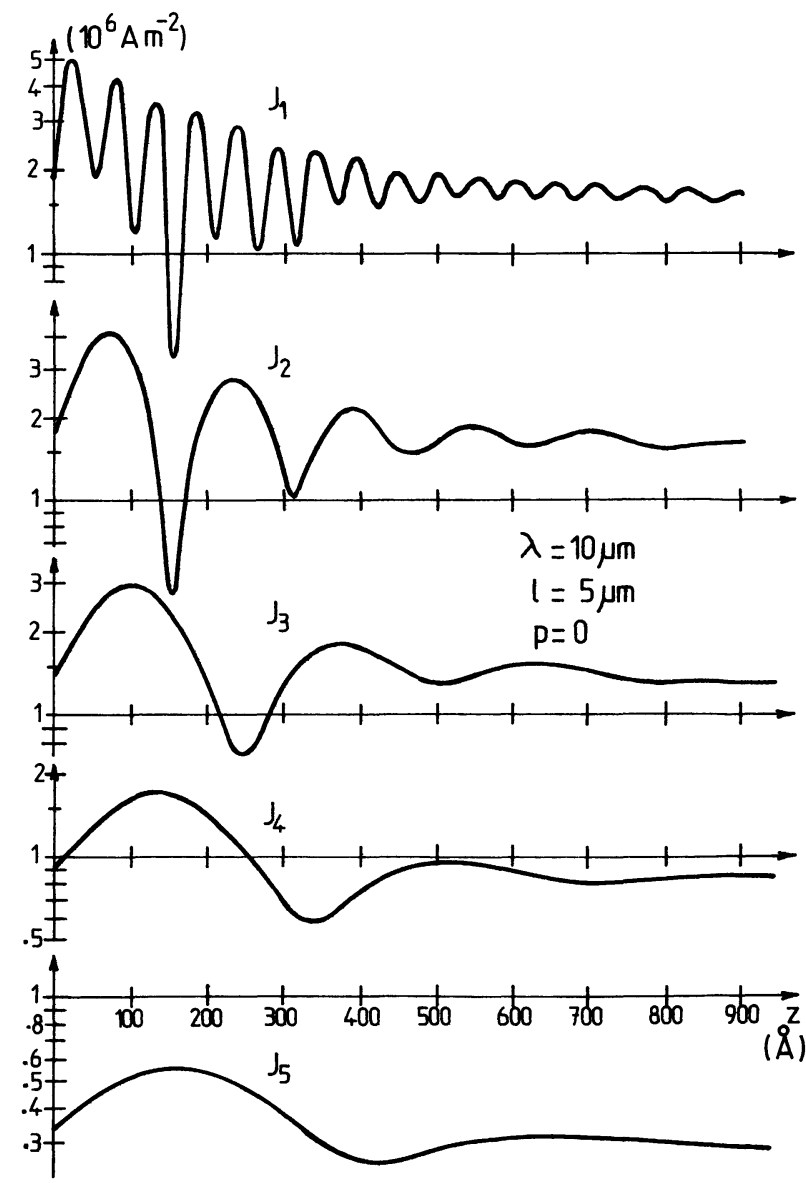

Fig. 13. - Variations spatiales de l'amplitude des composantes $J_{n}$ du courant de conduction. On a ici 5 composantes $(N=5), \lambda=10 \mu \mathrm{m}, l=5 \mu \mathrm{m}$, et $p=0$.

[Spatial variation of the components $J_{n}$ of the conduction current for the following parameter values $N=5, \lambda=10 \mu \mathrm{m}$ $l=5 \mu \mathrm{m}$ and $p=0$.] 
5.4 INFLUENCE DE LA POLARISATION DES IONS DU RÉSEAU. - La polarisation des ions du réseau se traduit par la présence du terme $k_{\mathrm{r}}=\varepsilon_{\mathrm{r}}^{1 / 2} \frac{\omega}{c}$ dans l'équation générale (19).

Ainsi que cela apparaît sur les figures 14 et 15 , l'influence de la constante diélectrique $\varepsilon_{\mathrm{r}}$ reste très faible aux longueurs d'onde de l'ordre de 1 à $2 \mu \mathrm{m}$; elle peut être considérée comme négligeable au-delà de $2 \mu \mathrm{m}$.

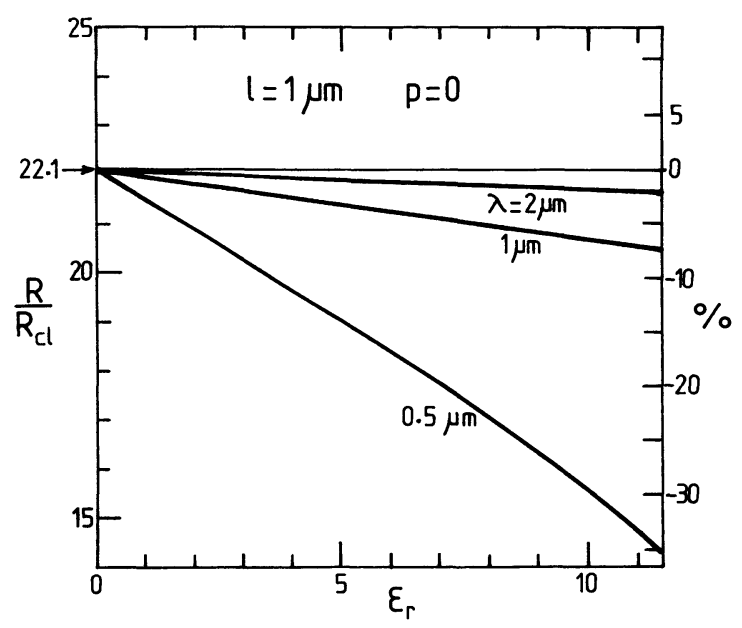

Fig. 14. - Influence de la constante diélectrique locale $\varepsilon_{\mathrm{r}}$ (considérée comme réelle) associée aux ions du réseau métallique, sur la résistance de surface. L'échelle verticale de droite représente l'écart en pourcentage par rapport à la valeur obtenue pour $\varepsilon_{\mathrm{r}}=0$. L'anomalie correspond à un libre parcours moyen $l=1 \mu \mathrm{m}$ intermédiaire entre les valeurs extrêmes obtenues à température ambiante $(l \simeq 0,03 \mu \mathrm{m})$ et la température de l'hélium liquide $(l \simeq 8$ à $10 \mu \mathrm{m}) . p$ a été choisi égal à 0 car dans le visible et l'infrarouge proche, la valeur $p=1$ conduit à des résultats pratiquement identiques à ceux de la théorie classique.

[Influence of the local lattice dielectric constant $\varepsilon_{\mathrm{r}}$ on the surface resistance $R_{\mathrm{s}}\left(\varepsilon_{\mathrm{r}}\right)$. The right vertical scale gives the difference between $R_{\mathrm{s}}\left(\varepsilon_{\mathrm{r}}\right)$ and $R_{\mathrm{s}}(0)$ for $p=0$. (In the visible and near IR, the condition $p=1$ gives results in good agreement with those of the classical theory.) The observed anomaly corresponds to a $l p m l=1 \mu \mathrm{m}$ that is an intermediate figure between values obtained at ambient helium temperature $(l \simeq 0.03 \mu \mathrm{m})$ and values obtained at liquid helium temperature $(l \simeq 8-10 \mu \mathrm{m})$.]

On a supposé ici que $\varepsilon_{\mathrm{r}}$ est réelle, c'est-à-dire que la fréquence de l'onde est loin de toute bande d'absorption. On remarque sur la figure 15 que les courbes obtenues à $\lambda=0,5$ et $1 \mu \mathrm{m}$ sont confondues si l'on fait $\varepsilon_{\mathrm{r}}=0$. La prise en compte de la constante diélectrique locale du réseau a donc pour effet de masquer l'effet de peu anormal dans le domaine visible, aux températures voisines de la température ambiante $(l \simeq 0,03 \mu \mathrm{m})$.

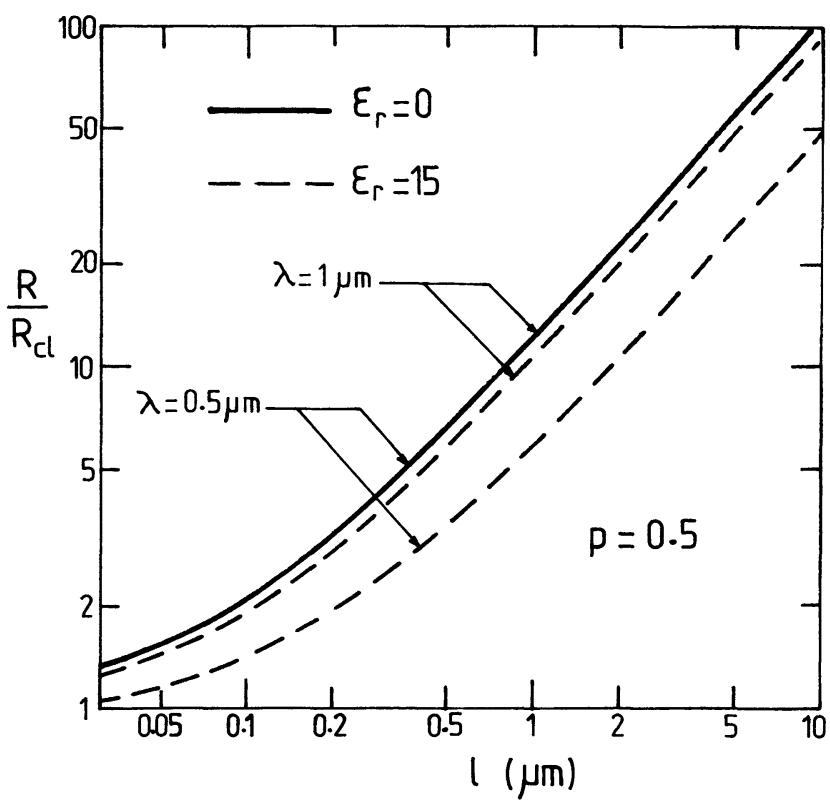

Fig. 15. - Variation de la résistance de surface avec le libre parcours moyen $l$ aux longueurs d'onde $\lambda=0,5$ et $1 \mu \mathrm{m}$ et pour $\varepsilon_{\mathrm{r}}=0$ (tracé continu) et $\varepsilon_{\mathrm{r}}=15$ (tracé interrompu). Les courbes correspondant à $\varepsilon_{\mathrm{r}}=0$ sont confondues.

[Variation of surface resistance as a function of mean free path $l$ at two wavelengths $(\lambda=0.5$ and $1 \mu \mathrm{m})$ for $\varepsilon_{\mathrm{r}}=0$ (continuous lines) and $\varepsilon_{\mathrm{r}}=15$ (interrupted lines). Both curves correspondind to $\varepsilon_{\mathrm{r}}=0$ are coincident.]

\section{Conclusion.}

Nous avons présenté une méthode de calcul numérique de l'impédance de surface basée sur les équations de la théorie de l'effet de peau anormal de Reuter et Sondheimer.

L'interprétation physique de ces équations nous a conduit, au $\S 2.2$, à définir des fonctions de distribution $\varepsilon(z, s)$ du champ électrique et $j(z, s)$ du courant de conduction, la variable $s=\sin \theta$ étant associée à tous les électrons dont la trajectoire fait l'angle $\theta$ avec la surface.

La représentation de $\varepsilon$ et $j$ par $N$ valeurs discrètes nous a ensuite permis de décomposer l'amplitude du champ électrique en $N+1$ ondes du type $a_{p} \exp \left(i u_{p} z\right)$ et d'en déduire l'impédance de surface $Z_{\mathrm{s}}$.

Contrairement à une approche purement mathématique faisant appel aux transformées de Fourier ou de Laplace, cette méthode ne nécessite qu'un faible nombre de points d'intégration, c'est-à-dire de composantes spectrales, pour obtenir une bonne image du champ au voisinage de la surface. Par exemple, aux longueurs d'onde inférieures à $200 \mu \mathrm{m}$ environ, la précision des calculs est tout à fait satisfaisante pour $N=4$ ou 5 .

Dans le cas extrême où $N=1$, on obtient encore une bonne approximation de l'impédance de surface aux longueurs d'onde de l'ordre du $\mu \mathrm{m}$. Le calcul 
explicite de $Z_{\mathrm{s}}$ fait dans ces conditions nous a permis de retrouver l'expression approchée habituelle :

$$
Z_{\mathrm{s}} \# R_{\mathrm{cl}}\left[1+(1-p) \frac{3}{8} k_{p} l\right]+i X_{\mathrm{cl}} .
$$

Nous avons, en outre fait les constatations suivantes :

- La partie réelle de l'impédance de surface est bien décrite, dans le domaine $2<\lambda<100 \mu \mathrm{m}$, par l'expression approchée :

$$
R \# p R_{1}+(1-p) R_{0} .
$$

- La précision de la relation approchée (41) proposée dans une précédente publication est de l'ordre de $5 \%$ à $\lambda=10 \mu \mathrm{m}$.

- Lorsque le paramètre $p$ n'est pas isotrope, on peut le remplacer par sa valeur moyenne $\langle p\rangle$ obtenue à partir de la fonction de pondération $w(s)$ définie au $\S 5.2$.

- La variation spatiale du champ électrique reste très proche d'une exponentielle pour $\lambda<10 \mu \mathrm{m}$. Cela justifie que l'on puisse, aux longueurs d'onde de l'ordre $\mathrm{du} \mu \mathrm{m}$, utiliser la théorie classique avec un temps de relaxation corrigé.

- Le courant de déplacement, considéré comme purement local, a une influence négligeable si $\lambda>2 \mu \mathrm{m}$.

Enfin, cette méthode est applicable au calcul de l'impédance de surface d'une couche mince.

Les composantes spectrales se dédoubleront alors en $a_{p}^{+} \exp \left(i u_{p} z\right)$ et $a_{p}^{-} \exp \left(-i u_{p} z\right)$.

Le mode de calcul des vecteurs d'onde propres $u_{p}$ restera inchangé car seules les amplitudes $a_{p}^{+}$dépendent des conditions aux limites.

\section{Annexe.}

CALCUl des COefFicients de l'éQuation CARACtéristique (32). - Le développement de l'équation (26) du § 3.1 donne :

$$
\begin{aligned}
\sum_{n} B_{n}\left[X^{N-1}+X^{N-2} S_{1}^{\neq n}+X^{N-3} S_{2}^{\neq n}+\cdots+X S_{N-2}^{\neq n}\right. & \left.+S_{N-1}^{\neq n}\right]= \\
& =\left(X-Q_{0}\right)\left[X^{N}+X^{N-1} S_{1}+X^{N-2} S_{2}+\cdots+S_{N}\right] .
\end{aligned}
$$

La fonction $\S_{p}$ est définie au § 3.1 par (34).

La fonction $S_{p}^{\neq n}$ représente la somme de tous les produits possibles de $p$ termes du type $\alpha_{i}^{2}$ avec $i \neq n$. Il y a donc $N-1$ termes à considérer.

On obtient par identification à (32) les coefficients suivants :

$$
\begin{aligned}
C_{1} & =1 \\
C_{2} & =-Q_{0}+\delta_{1} \\
C_{3} & =-Q_{0} \delta_{1}+\delta_{2}-\sum_{n=1}^{N} B_{n} \\
\hdashline=4 \mathrm{à} N+1 \quad C_{p} & =-Q_{0} \delta_{p-2}+\delta_{p-1}-\sum_{n=1}^{N} B_{n} s_{p-3}^{\neq n} \\
C_{N+2} & =-Q_{0} \delta_{N}-\sum_{n=1}^{N} B_{n} \delta_{N-1}^{\neq n} .
\end{aligned}
$$

Entre les fonctions du type $S_{p}$ et $S_{p}^{\neq n}$ on peut établir la relation de récurrence suivante :

$$
S_{p}^{\neq n}=S_{p}-\alpha_{n}^{2} S_{p-1}^{\neq n} .
$$

Cela permet d'exprimer les coefficients précédents exclusivement à partir de fonctions du type $S_{p}$ car on peut écrire :

$$
\sum_{n=1}^{N} B_{n} \mathcal{S}_{p}^{\neq n}=\mathcal{S}_{p} \sum_{n} B_{n}-\mathcal{S}_{p-1} \sum_{n} B_{n} \alpha_{n}^{2}+\delta_{p-2} \sum_{p} B_{n} \alpha_{n}^{4}+\cdots+(-1)^{p-1} \mathcal{S}_{1} \sum_{n} B_{n} \alpha_{n}^{2(p-1)}+(-1)^{p} \sum_{n} B_{n} \alpha_{n}^{2 p}
$$

Désignant cette suite par $\mathcal{L}_{p}$, on obtient bien la relation (36) :

$$
\mathfrak{L}_{p}=S_{p} \mathcal{M}_{1}+S_{p-1} \mathcal{M}_{2}+S_{p-2} \mathscr{M}_{3}+\cdots+S_{1} \mathcal{M}_{p}+\mathcal{M}_{p+1}
$$

dans laquelle $\mathcal{H}_{p}$ est définie par la formule (35). 
MÉTHODE DE CALCUL DES $S_{p}$. - Si l'on représente chaque élément $\alpha_{i}^{2}$ par son indice $i$, l'ensemble des calculs à effectuer peut être symbolisé par un graphe qui, pour $N=5$, aura l'allure ci-dessous,


5

Chaque liaison entre deux indices doit être considérée comme une multiplication.

On peut définir sur une même ligne horizontale de numéro $p$ un ensemble de $C_{N}^{p}$ termes que l'on notera $\mathrm{A}_{p}^{n}\left(n=1\right.$ à $\left.\mathrm{C}_{N}^{p}\right)$, chacun d'eux étant le produit de tous les $\alpha_{i}^{2}$ dont les indices sont sur le chemin permettant de remonter à la première ligne.

On écrira par exemple :

$$
\begin{aligned}
\delta_{3} & =A_{3}^{1}+A_{3}^{2}+\cdots+A_{3}^{10} \\
& =123+124+125+134+135+\cdots+345 .
\end{aligned}
$$

Un terme tel que 124 doit être compris comme le produit $\alpha_{1}^{2} \alpha_{2}^{2} \alpha_{4}^{2}$. Comme on a besoin des valeurs de toutes les fonctions $\delta_{p}$ pour $p=1$ à $N$, on a intérêt à réduire le nombre de calculs en utilisant la relation :

$$
\mathrm{S}_{N-p}=\left(\sum_{n=1}^{\mathrm{C}_{N}^{p}} \frac{1}{\mathrm{~A}_{p}^{n}}\right) \prod_{i=1}^{N} \alpha_{i}^{2} .
$$

Cela permet de ne calculer qu'environ la moitié des $\mathrm{A}_{p}^{n}$.

ERREUR DE TRONCATURE. - L'expression du courant sous sa forme discrète est la suivante :

$$
J(z)=\frac{3}{4} \frac{\sigma}{l} \sum_{i=1}^{N} \frac{1-s_{i}^{2}}{s} \Delta_{i} \mathfrak{F}\left(s_{i}, z\right) .
$$

L'erreur de troncature peut être estimée en cherchant l'expression correspondant à une très faible anomalie et $p=1$.

Compte tenu de la relation $(10)$ du $\S 2.1$, on peut écrire :

$$
J(z) \# \frac{3}{2} \frac{\sigma}{1-i \omega \tau} E(z) \sum_{i=1}^{N}\left(1-s_{i}^{2}\right) \Delta_{i} \# \frac{\sigma}{1-i \omega \tau} E(z) .
$$

L'erreur de troncature aura donc un ordre de grandeur donné par l'écart :

$$
\frac{3}{2} \sum_{i=1}^{N}\left(1-s_{i}^{2}\right) \Delta_{i}-1
$$

Dans les conditions des calculs dont les résultats sont présentés au chapitre 5, on trouve pour $N=4$ une erreur de 0,008 . 


\section{Bibliographie}

[1] Reuter, G. E. H. and Sondheimer, E. H., Proc. $R$. Soc. A 195 (1948) 336.

[2] Dingle, R. B., Appl. Sci. Res. B 3 (1953) 69.

[3] Dingle, R. B., Physica XIX (1953) 311.

[4] Drangeid, K. E. and Sommerhalder, R., Helv. Phys. Acta 48 (1975) 561.

[5] Gilberd, P. W., J. Phys. F 12 (1982) 845.

[6] TheYe, M. L., Phys. Lett. 25A (1967) 764.

[7] Beach, R. T. and Christy, R. W., Phys. Rev. B 16 (1977).

[8] Holstein, T., Phys. Rev. 88 (1952) 1427.

[9] Theye, M. L., Phys. Rev. B 2 (1970) 3060.

[10] Schlesinger, Z. and Sievers, A. J., Solid State Commun. 43 (1982) 671.
[11] ANGot, A., Compléments de Mathématiques (Masson) 1972.

[12] Nowakowski, C., Méthodes de calcul numérique, éditions du P.S.I. (1982).

[13] Ralston, A. and Wilf, H. S., Méthodes Mathématiques pour calculateurs arithmétiques (Dunod, Paris) 1965.

[14] Peterseim, J., Thummes, G., Mende, H. H., Phys. Status Solidi 59 (1980) K-25.

[15] Dingle, R. B., Physica XIX (1953) 348.

[16] Dudek, J. C., Revue Phys. Appl. 19 (1984) 287. 